\title{
ARTICLE
}

\section{A transposable element in a NAC gene is associated with drought tolerance in maize seedlings}

Hude Mao ${ }^{1,2}$, Hongwei Wang ${ }^{1,2}$, Shengxue Liu' ${ }^{1}$ Zhigang $\mathrm{Li}^{1}$, Xiaohong Yang ${ }^{3}$, Jianbing Yan ${ }^{4}$, Jiansheng $\mathrm{Li}^{3}$, Lam-Son Phan $\operatorname{Tran}^{5} \&$ Feng Qin ${ }^{1}$

Drought represents a major constraint on maize production worldwide. Understanding the genetic basis for natural variation in drought tolerance of maize may facilitate efforts to improve this trait in cultivated germplasm. Here, using a genome-wide association study, we show that a miniature inverted-repeat transposable element (MITE) inserted in the promoter of a NAC gene ( $Z m N A C 111)$ is significantly associated with natural variation in maize drought tolerance. The 82-bp MITE represses ZmNAC111 expression via RNA-directed DNA methylation and H3K9 dimethylation when heterologously expressed in Arabidopsis. Increasing ZmNAC111 expression in transgenic maize enhances drought tolerance at the seedling stage, improves water-use efficiency and induces upregulation of drought-responsive genes under water stress. The MITE insertion in the ZmNAC111 promoter appears to have occurred after maize domestication and spread among temperate germplasm. The identification of this MITE insertion provides insight into the genetic basis for natural variation in maize drought tolerance.

\footnotetext{
${ }^{1}$ Key Laboratory of Plant Molecular Physiology, Institute of Botany, Chinese Academy of Sciences, Beijing 100093, China. ${ }^{2}$ Graduate University of the Chinese Academy of Sciences, Beijing 100049, China. ${ }^{3}$ National Maize Improvement Center of China, China Agricultural University, Beijing 100193 , China. ${ }^{4}$ National Key Laboratory of Crop Genetic Improvement, Huazhong Agricultural University, Wuhan 430070, China. ${ }^{5}$ Signaling Pathway Research Unit, RIKEN Center for Sustainable Resource Science, 1-7-22 Suehiro-cho, Tsurumi-ku, Yokohama 230-0045, Japan. Correspondence and requests for materials should be addressed to F.Q. (email: qinfeng@ibcas.ac.cn).
} 
M aize (Zea mays) production is frequently compromised by water scarcity, which is aggravated by trends of climate warming and erratic rainfall patterns on a global scale ${ }^{1,2}$. During the past two decades, although progress has been made towards an overall increase in maize yields, plant sensitivity to drought stress has increased ${ }^{1}$. It is proposed that improving the ability of maize to overcome stress rather than primary productivity could be a primary contributor in efforts to achieve higher yielding maize ${ }^{3}$. Thus, enhanced drought tolerance has become a priority trait in current maize genetic improvement efforts. However, the identification of the genetic components underlying drought tolerance has proven to be challenging. To date, no quantitative trait loci (QTLs) responsible for maize drought tolerance have been cloned, despite the reports of their mapping information ${ }^{4-7}$.

Traditional QTL mapping, based on the genetic linkage of a certain trait with the molecular marker within a bi-parental segregation population, usually recombinant inbred lines (RILs), has been successfully used to identify the genes underlying QTLs for glume architecture ${ }^{8}$, branched architecture ${ }^{9}$, flowering time $e^{10}$, photoperiod sensitivity ${ }^{11}$, resistance to head smut ${ }^{12}$ and so on. However, it usually takes a relatively long time to generate RILs of the two parents, which are phenotypically contrasting with respect to the trait. Moreover, the mapping resolution is largely dependent on the genetic recombination among these RILs. To achieve accurate mapping and gene cloning, it usually requires either a large number of RILs initially or several consecutive steps of selfing or backcrossing, to narrow down the region containing the candidate gene. Thus, a large number of QTL mapping studies have been conducted but only a limited number of them were cloned ${ }^{13}$. Another limitation of this approach is that the functional variation(s) of only two parental alleles can typically be evaluated during the mapping process.

More recently, genome-wide association study (GWAS), which is based on genetic linkage disequilibrium (LD) and makes use of natural variation and recombinants, has been used as a novel strategy for dissecting complex trait loci in plants ${ }^{14-17}$. Since historical recombinant events can be exploited in a collection of a large number of genotypes, LD mapping can reach a high resolution and investigate multiple alleles of a single locus ${ }^{14}$. With the development of high-throughput DNA variation discovery technology and improvement of statistical analyses, GWAS has gained favourability in genetic research in various plant species. Especially, due to the rapid LD decay in the maize genome, GWAS has facilitated the genetic dissection of several complex traits, including kernel $\beta$-carotene ${ }^{18}$ and oil content ${ }^{19}$, and flowering time in maize ${ }^{20}$. Although association studies of maize drought tolerance have been attempted ${ }^{21-24}$, the proposed candidate genes or their causative variations still remain to be verified and resolved.

In the maize genome, $\sim 85 \%$ of the genomic contents are composed of transposable elements (TEs), and the generic sequences are embedded in a vast expanse of $\mathrm{TEs}^{25}$. To maintain stability of the genome, TEs are usually silenced and inactive, due to DNA and chromatin modifications ${ }^{26}$. However, TEs have been shown to play important roles in plant evolution and environmental adaptation. For instance, a hopscotch element inserted at $\sim 60-\mathrm{kb}$ upstream of teosinte branched 1 ( $t b 1)$ increased maize apical dominance ${ }^{9}$ and a CACTA-like TE located $\sim 2-\mathrm{kb}$ upstream of ZmCCT was found to contribute to maize photoperiod sensitivity ${ }^{20}$. Miniature inverted-repeat transposable elements (MITEs) are a kind of non-autonomous DNA transposon, which are usually shorter than $600 \mathrm{bp}$ and widespread in plant genomes ${ }^{27}$. A MITE insertion in $\sim 70-\mathrm{kb}$ upstream of $Z m R A P 2.7$ was demonstrated to be associated with maize flowering time ${ }^{10}$. TEs can influence nearby gene expression either through the cis-acting element residing in their own sequences, or by changing the DNA or chromatin methylation status of adjacent genes ${ }^{26,28,29}$. In rice (Oryza sativa), MITEs have been recently discovered to be capable of generating 24-nt small interfering RNA (siRNA), depending on Dicer-like 3a (OsDCL3a) activity, and interfering nearby gene expression through RNA-directed DNA methylation $(\mathrm{RdDM})^{30}$. In plants, the RdDM pathway consists of the following major steps: (1) the RNA polymerase IV transcribes single-strand RNAs from repetitive heterochromatin regions; (2) its physically associated RNA-dependent RNA polymerase 2 (RDR2) synthesizes the double-stranded RNA (dsRNAs); (3) the dsRNAs are cleaved by DCL3 into 24-nt siRNAs; and (4) ARGONAUTE 4 (AGO4) subsequently loads the siRNAs to their complementary DNA regions. Lastly, the formed complex recruits the DNA methyltransferase (DRM2) to catalyse methylation at cytosine in $\mathrm{CG}, \mathrm{CHG}$ and $\mathrm{CHH}$ contents $(\mathrm{H}=\mathrm{A}, \mathrm{G}$ or $\mathrm{C})$, especially in $\mathrm{CHH}$ sequence context, which is a hallmark of $\mathrm{RdDM}^{31-33}$. Enrichment of chromatin histone3 lysine 9 dimethylation (H3K9me2), which is mainly catalysed by a histone methyltransferase, SUVH4 (also named KYP), couples the DNA methylation in the adjacent regions ${ }^{30,34}$.

Here, we show by GWAS that an 82-bp (MITE) insertion in the promoter region of a NAC gene $(Z m N A C 111)$ is associated with maize drought tolerance. The MITE insertion correlates with lower $Z m N A C 111$ expression in maize, and when heterologously expressed in Arabidopsis it suppresses $Z m N A C 111$ expression via the RdDM pathway. Transgenic studies demonstrated that enhanced expression of $\mathrm{ZmNAC111}$ conferred drought tolerance in both transgenic Arabidopsis and maize seedlings, improves plant water-use efficiency (WUE) and enhances the expression of stress-responsive genes under the stress. A comparison of MITE insertion frequency and nucleotide diversity at the $\mathrm{ZmNAC111}$ locus among teosinte, tropical/subtropical (TST) and temperate genotypes suggests that the MITE insertion appears to have occurred after maize domestication from teosinte and spread in the temperate germplasm.

\section{Results}

ZmNAC111 is associated with maize seedling drought tolerance. To identify genes associated with maize drought tolerance, we performed GWAS by analysing a natural maize population, consisting of 368 inbred lines that were collected from TST and temperate regions of the world ${ }^{19}$. Approximately, 560,000 single nucleotide polymorphisms (SNPs) ${ }^{19}$ were applied to the study. Considering the complexity of plant drought tolerance, which is affected by both the time period and intensity of the stress imposed to plants, we decided to focus on tolerance to severe drought stress at the seedling stage. Seedling survival rate (SR) can reflect plant tolerance mechanisms and cellular responses to drought. It is less affected by environmental fluctuation, which helps to identify of the underlying genetic determinant(s). The drought tolerance of each genotype was assayed by calculating an SR index (percentage of the survived plants after re-watering) under severe drought stress at seedling stage ${ }^{24}$. On average, the inbred lines from TST exhibited higher SR in comparison with those from temperate regions, indicating that maize germplasm derived from areas near to the place of origin may be more drought tolerant than those cultivated in temperate regions. On the whole-genome scale, in consideration of the population structure $(\mathrm{Q})$ and parental relatedness $(\mathrm{K})$ of the population, a SNP within GRMZM2G127379 on chromosome 10 was identified to be significantly associated with plant drought tolerance (Supplementary Fig. 1). GRMZM2G127379 encodes a NAC-type transcription factor (TF), belonging to a family with more than 
100 members in maize genome. GRMZM2G127379 has previously been designated as ZmNAC111 (ref. 35). Phylogenetic analysis of the amino acid sequence encoded by ZmNAC111 indicated that its closest identified homologous gene in rice is OsNAC10 (LOC_Os11g03300) $^{36}$ (Supplementary Fig. 2).

NAC proteins regulate multiple biological processes in plants, including cotyledon ${ }^{37}$ and root development ${ }^{38}$, formation of secondary walls ${ }^{39}$, leaf senescence ${ }^{40}$, nutrient remobilization to grains $^{41}$ and stress responses ${ }^{42}$. Considering the possible function of NAC-type genes in plant drought tolerance, we then sequenced the $Z m N A C 111$ gene in 262 maize inbred lines. A 2.3-kb genomic region, spanning the $5^{\prime}$ - to $3^{\prime}$-untranslation region (UTR) of ZmNAC111, was analysed. A total of 157 SNPs and 119 InDels (insertions and deletions) were further identified. A newly identified 82-bp InDel (InDel-572), located 572-bp upstream of the start codon of ZmNAC111, was found to have the greatest significant association with the seedling SR $\left(P=5.52 \times 10^{-6}\right.$; calculated under mixed linear model, see Methods; Fig. 1a), contributing to $7.27 \%$ of the phenotypic variation in the natural population. Two non-synonymous variations in exon3 were also identified as marginally significant (Supplementary Data 1). SNP1532 resulted in an amino acid residue change of proline (Pro) into glutamine (Gln), and SNP1535 resulted in an alteration of Gln to arginine (Arg). Both mutations, which were in strong $\mathrm{LD}$, locate in the $\mathrm{C}$-terminal transcriptional regulatory region but not in the N-terminal DNA-binding domain of the ZmNAC111 protein. InDel-572 was in LD with the variations in $5^{\prime}$-UTR and the first exon $\left(r^{2}>0.4\right)$, but not with the two non-synonymous SNPs (Fig. 1a). All the other variations were not associated to the trait with statistical significance.

InDel-572 is a MITE insertion in the ZmNAC111 promoter. Sequence analysis of InDel-572 in the promoter region of ZmNAC111 gene revealed that it is composed of a long-terminal inverted repeat (38-bp for each), a 4-bp loop and two additional nucleotides 'TA' at the end. Another 'TA' sequence was found directly before this insertion (Fig. 1b). It represents a typical structure of a MITE insertion within the genome, which is usually short with approximately hundreds of bps and consists of TIRs and target-site direct repeats, with a preferential insertion at TA or TAA ${ }^{27}$. We blasted the maize TE database using the 82-bp sequence as a query (http://maizetedb.org/ maize/) and found that it belongs to the Tc1/Mariner superfamily of MITEs. The 80-bp DNA sequence, excluding the target-site direct repeat, can form a perfect stem-loop structure (Fig. 1b). It is present in the promoter of ZmNAC111 of drought-sensitive genotypes, such as B73 and Mo17, whereas it is absent in the drought-tolerant genotypes, such as CIMBL55, 92, 70 and CML118 (Fig. 1b).

The MITE insertion correlates with lower ZmNAC111 expression. Since the MITE insertion locates in the promoter region of $Z m N A C 111$, we hypothesized that it results in altered ZmNAC111 expression among different genotypes. To examine this hypothesis, we analysed the expression of the ZmNAC111 gene among 133 inbred lines under well-watered, moderate and severe drought conditions. Quantitative reverse-transcription PCR (qRT-PCR) analysis of a total of 399 RNA samples revealed that ZmNAC111 expression positively correlated with plant SR under both moderate and severe drought stresses, but not under well-watered conditions (Fig. 2a; Supplementary Data 2). This finding suggested that increased expression of ZmNAC111 might contribute to the drought tolerance of natural maize varieties examined in this study under water stress. Furthermore, the qRT-PCR result revealed that regardless of the levels of stress imposed, the genotypes without the MITE $\left(\mathrm{MITE}^{-}\right)$had a

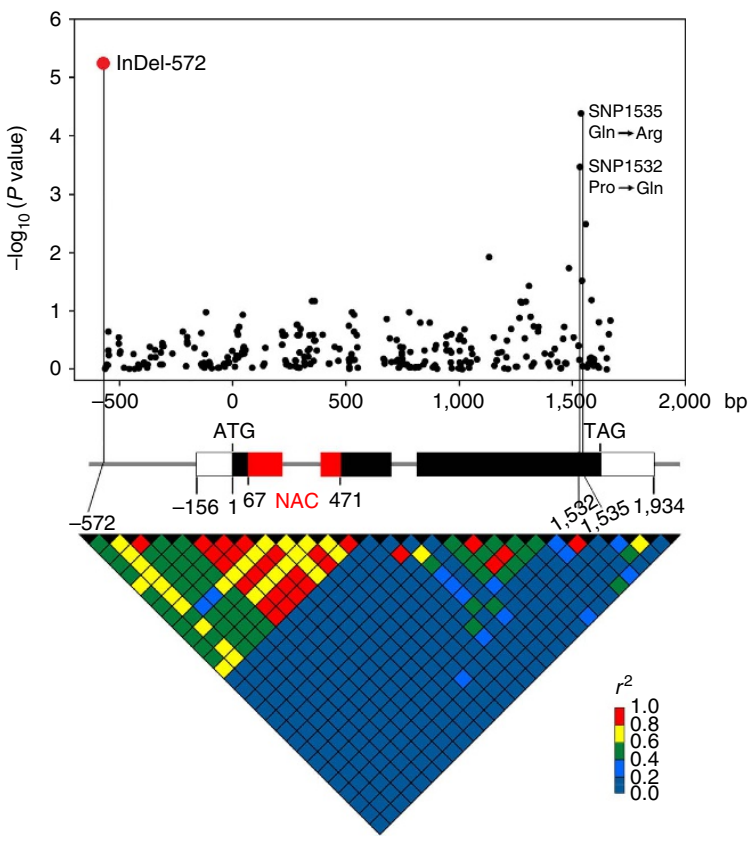

b

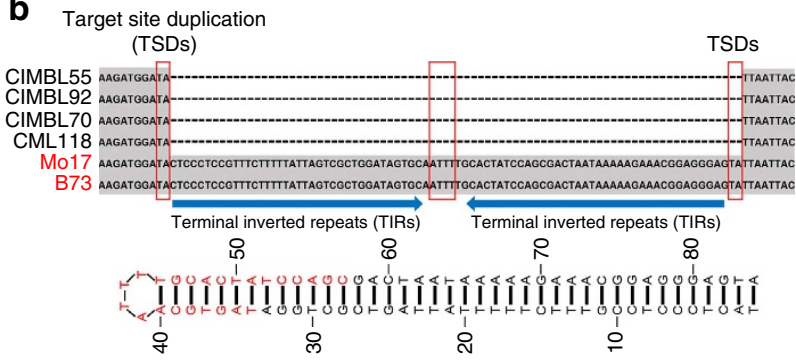

Figure 1 | An 82-bp MITE insertion in the ZmNAC111 promoter associated with maize drought tolerance. (a) The association analysis of genetic variation in ZmNAC111 with drought tolerance in maize and the pattern of pairwise LD of DNA polymorphisms. A schematic diagram of the $2.3-\mathrm{kb}$ genomic region of ZmNAC111, including the $5^{\prime}-, 3^{\prime}-U T R$, three exons and two introns is presented. The location of the start codon (ATG) is labelled as $'+1$ '. The region encoding the NAC domain is indicated in red. The most significant InDel (InDel-572) in the promoter and two non-synonymous variations in the coding region are connected to their locations in the gene diagram by solid lines. SNP1532 produced a change of Pro to Gln in the encoded protein, and SNP1535 changed GIn to Arg. (b) The DNA sequence and structure of the 82-bp MITE inserted in the ZmNAC111 promoter. The target site duplications (TSDs) and the loop are indicated by the red boxes. The blue arrows indicate two terminal inverted repeats. The predicted hairpin structure of the MITE is illustrated at the bottom. A 24-nt siRNA sequence found in the Cereal Small RNA Database that aligned to the MITE is highlighted in red. The MITE is present in the promoter of the ZmNAC111 gene in many of the drought-sensitive genotypes, such as B73 and Mo17, whereas it is absent in drought-tolerant genotypes, such as CIMBL55, 92, 70 and CML118.

significantly higher expression of ZmNAC111 than those with the MITE insertion (MITE ${ }^{+}$; Fig. 2b). The contrasting phenotype of drought tolerance and the differential ZmNAC111 expression of several representative genotypes were shown (Supplementary Fig. 3). On the basis of these data, we suggest that the MITE insertion may repress $Z m N A C 111$ expression resulting in higher sensitivity of $\mathrm{MITE}^{+}$maize varieties to drought stress.

To further test the association between the MITE insertion at the ZmNAC111 locus and seedling drought tolerance, we constructed three bi-parental $\mathrm{F}_{2: 3}$ populations. The genotypes of 
a

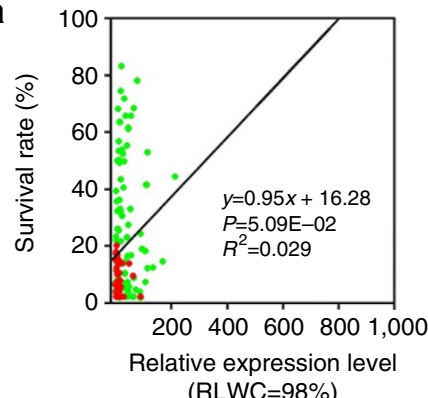

(RLWC=98\%)

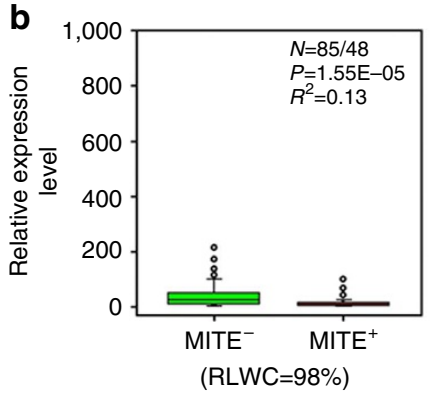

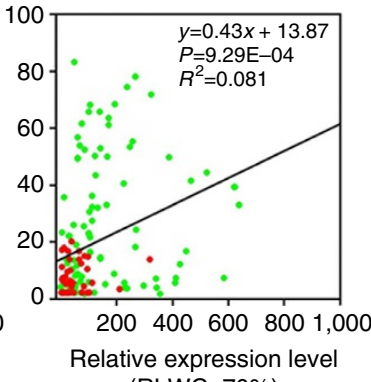

(RLWC=70\%)

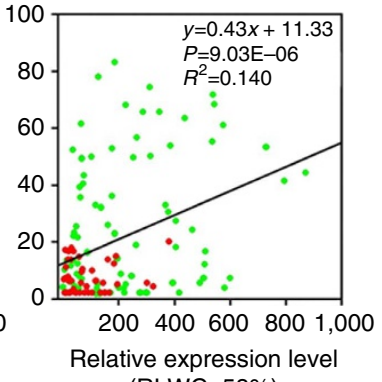

(RLWC=58\%)
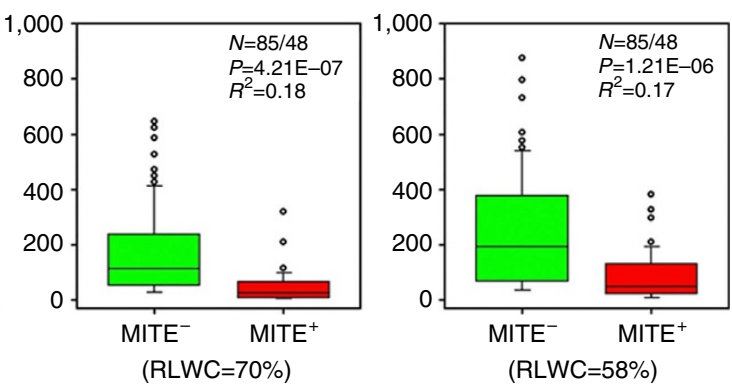

Figure 2 | Expression level of ZmNAC111. Relative expression level of ZmNAC111 under well-watered, moderate and severe drought conditions in relation to the rate of plant survival, and the presence or absence of the MITE (InDel-572) insertion. (a) Correlation of plant survival rate with the relative expression level of ZmNAC111. Drought stress was estimated by the decrease in the relative leaf water content (RLWC) from 98\% (well watered) to 70\% (moderate drought) and to $58 \%$ (severe drought). The red and green dots represent genotypes with or without the MITE insertion (MITE ${ }^{+}$and MITE $^{-}$) in the

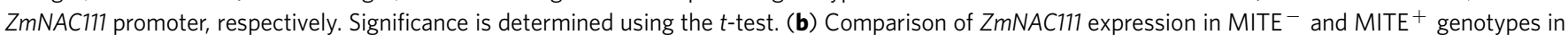
relation to RLWC. One-way analysis of variance (ANOVA) is applied to determine statistical differences in ZmNAC111 expression.

two parents of each population were either MITE ${ }^{+}$or MITE $^{-}$at the ZmNAC111 locus. We observed that the $\mathrm{MITE}^{-}$allele cosegregated with drought tolerance in three bi-parental $\mathrm{F}_{2: 3}$ populations (Supplementary Fig. 4). On the other hand, when we compared the transactivation activity of ZmNAC111 proteins encoded by alleles that differed in the two non-synonymous variations, SNP1532 and SNP1535, we found that these two variations in different alleles did not significantly affect the transactivation activity of the ZmNAC111 (Supplementary Fig. 5). Collectively, on the basis of our results, we suggest that the MITE insertion is the likely cause for differences in drought tolerance associated with $Z m N A C 111$, rather than amino acid changes in the encoded protein, and that the MITE ${ }^{+}$is the drought-sensitive and MITE $^{-}$is the drought-tolerant allele of ZmNAC111.

Correlation with DNA and histone methylation differences. To understand why the 82-bp MITE insertion may be correlated with reduced $Z m N A C 111$ expression, we checked the DNA and histone methylation status of ZmNAC111 in the MITE ${ }^{-}$and $\mathrm{MITE}^{+}$genotypes. Eight regions (R1-R8) spanning the $\mathrm{ZmNAC111}$ gene and promoter in two inbred lines, B73 (drought sensitive, MITE ${ }^{+}$) and CIMBL55 (drought tolerant, MITE ${ }^{-}$), were representatively analysed. Results revealed that only R1 and $\mathrm{R} 2$, nearest the MITE insertion, were hypermethylated in ZmNAC111-B73 but not in ZmNAC111-CIMBL55, regardless of the stress treatment (Fig. 3a). Bisulphite sequencing of these regions detected DNA hypermethylation, especially at the $\mathrm{CHH}$ content (Fig. 3b). Moreover, chromatin immunoprecipitation (ChIP) using a specific H3K9me2 antibody and followed by qPCR analysis demonstrated that $\mathrm{H} 3 \mathrm{~K} 9 \mathrm{me} 2$ was significantly enriched in $\mathrm{R} 1-\mathrm{R} 4$ in $Z m N A C 111-B 73$ in comparison with that in

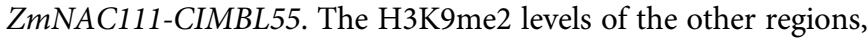
however, remained comparable. On the basis of these data, we suggest that the MITE insertion represses $Z m N A C 111$ expression through DNA and histone methylation of its nearby regions (Fig. 3c,d).

Repression of ZmNAC111 expression through RdDM pathway. A considerable number of 21- to 24-nt siRNA species were identified that could be aligned to the MITE sequence in the maize small RNA database (http://sundarlab.ucdavis.edu/smrnas/; Supplementary Fig. 6). In an attempt to check the hypothesis that the 82-bp MITE insertion may mediate DNA and histone methylation through the $\mathrm{RdDM}$ pathway, the genomic fragments of $g Z m N A C 111-B 73$ and $g Z m N A C 111-C I M B L 55$ were transformed into Arabidopsis and expressed with the CaMV $35 \mathrm{~S}$ promoter (Fig. 4a). The resultant 48 independent $\mathrm{T}_{2}$ transgenic lines for each construct were analysed. The transgenic plants harbouring 35S:gZmNAC111-CIMBL55 generally exhibited significantly higher levels of $Z m N A C 111$ expression, compared with the 35S:gZmNAC111-B73 transgenics (Fig. 4b). DNA hypermethylation and $\mathrm{H} 3 \mathrm{~K} 9 \mathrm{me} 2$ enrichment in the region nearby the MITE insertion were expectedly observed in the 35S:gZmNAC111-B73, but not in 35S:gZmNAC111-CIMBL55 transgenic lines (Fig. 4c-e). Plant drought tolerance was then compared between these two types of transgenics. The transgenic Arabidopsis harbouring 35S:gZmNAC111-CIMBL55 displayed greater drought tolerance than those transformed with 35S:gZmNAC111-B73 (Supplementary Fig. 7).

To verify that the MITE insertion-mediated ZmNAC111 repression through the $\mathrm{RdDM}$ pathway when heterologously expressed in Arabidopsis, five mutants defective in key genes involved in the $\mathrm{RdDM}$ pathway were crossed with two independent homozygous 35S:gZmNAC111-B73-2 and -23 lines to transfer the 35S:gZmNAC111-B73 construct to the RdDMdefective backgrounds. In the drm1-2;drm2-2, ago4-5 and rdr2-2 mutants, $Z m N A C 111-B 73$ expression was greatly enhanced, while its DNA methylation and $\mathrm{H} 3 \mathrm{~K} 9 \mathrm{me} 2$ were reduced to a comparable level relative to those of the $Z m N A C 111-C I M B L 55$ in the wild-type 

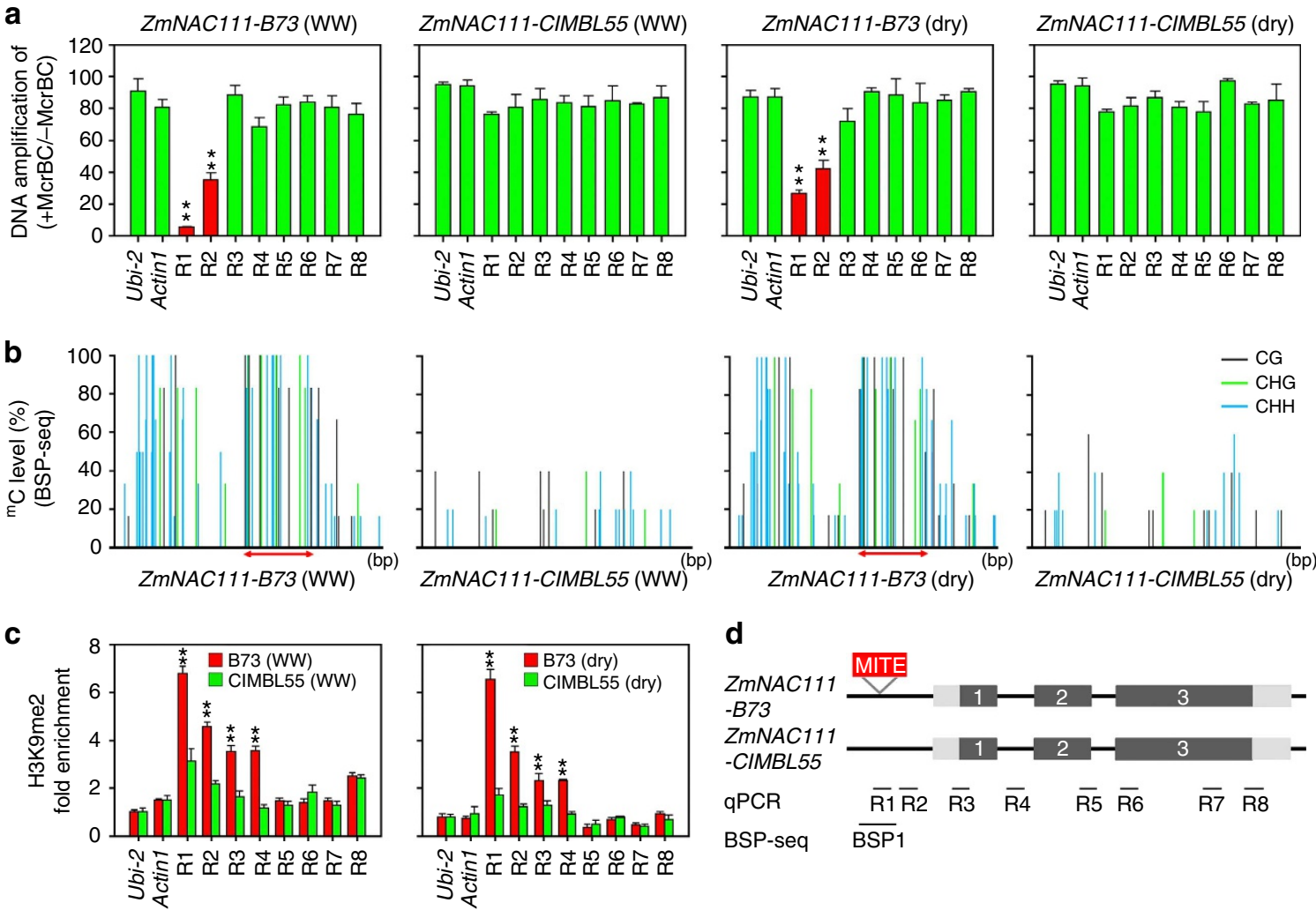

Figure 3 | DNA and H3K9me2 methylation status of the drought-tolerant and drought-sensitive alleles of ZmNAC111. (a) DNA methylation status was determined by treatment with McrBC, a methylation-sensitive endonuclease followed by $\mathrm{qPCR}$ (McrBC-qPCR) analyses in the eight regions (R1-R8) of the genomic sequence of ZmNAC111. Genomic DNA was extracted from B73 and CIMBL55 genotypes grown under well-watered (WW) and moderate drought (dry, RLWC $=70 \%$ ) conditions. (b) Methylation of cytosine residues in CG, CHG and CHH sites (grey, green and blue lines, respectively) was revealed by bisulphite sequencing of the BSP1 region. The DNA samples of (a) were analysed. The MITE region is indicated by a double-sided arrow. (c) Chromatin state was detected using an anti-H3K9me2 ChIP-qPCR assay at eight different regions (R1-R8) of the genomic regions of ZmNAC111-B73 and ZmNAC111-CIMBL55. The H3K9me2 state of the maize Ubi2 and Actin1 were tested in parallel as negative controls. Anti-H3 was used as an internal reference in the ChIP-qPCR assay. (d) The positions of R1-R8 in the genomic region of ZmNAC111. The 5'- and 3'-UTR regions (light grey boxes), exons (grey boxes) and 82-bp MITE insertion (red box) are illustrated. Black lines indicate the position of the McrBC-qPCR, ChIP-qPCR and bisulphite sequencing (BSP1) analyses. Error bars are s.d. and significant differences are determined using the $t$-test, ${ }^{\star \star} P<0.01$.

background. In the suvh4-3 mutant, the H3K9me2 level of ZmNAC111-B73 was significantly reduced, but the DNA hypermethylation remained relatively similar to that in the wild-type background, which was consistent with the fact that the SUVH4 functions as a histone methyltransferase. In $d c l 2-1 ; d c l 4-2$, the gene expression, DNA methylation and $\mathrm{H} 3 \mathrm{~K} 9 \mathrm{me} 2$ levels of ZmNAC111-B73 still differed from those observed for the ZmNAC111-CIMBL55 in wild-type background, most likely due to the intact DCL3 function in this mutant (Fig. 4f-i). No remarkable change in gene expression, DNA methylation and H3K9me2 level was observed when 35S:gZmNAC111-CIMBL55-5 and -12 transgenic Arabidopsis plants were crossed with the RdDM mutants (Supplementary Fig. 8). Collectively, these data clearly indicated that MITE represses the ZmNAC111 expression through the RdDM pathway when heterologously expressed in Arabidopsis.

ZmNAC111 overexpression confers seedling drought tolerance. Given that ZmNAC111 expression is positively correlated with maize drought tolerance, we generated both transgenic Arabidopsis and maize, overexpressing the coding sequence of ZmNAC111 (from the B73 genotype). For the transgenic Arabidopsis, the phenotypes of three independent 35S:ZmNAC111 lines were analysed. In comparison with the empty-vector transformed plants (VC), the transgenic Arabidopsis displayed significantly enhanced drought tolerance, without remarkable morphological changes under normal growth conditions. When the survival of VC was around $20 \%, \sim 80 \%$ of the transgenic plants were alive in the parallel water-withholding experiments (Supplementary Fig. 9a-c). The transgenic Arabidopsis plants were also hypersensitive to exogenous abscisic acid (ABA) as shown by seed germination and stomatal closure assays, indicating an enhancement of ABA signalling in the transgenic plants (Supplementary Fig. 9e-g).

Similar improved drought tolerance was also observed in pot-grown transgenic maize transformed by ZmUbi:ZmNAC111. Under drought stress, $\sim 80 \%$ of the $\mathrm{T}_{2}$ generation transgenic maize plants survived; whereas, the SR of the transgenic-negative sibling plants (WT) was only $30 \%$ (Fig. 5c,d). No evident abnormal changes were observed in the transgenic maize compared with WT under normal growth conditions, although we acknowledge that these growth conditions likely do not accurately represent field conditions (Fig. 5a,b; Supplementary Data 3). Next, we compared the stomatal response and transpiration under progressive water stress between the transgenic maize and WT. The leaf photosynthesis rates (PS), stomatal conductance (SC) and transpiration rates (TR) were recorded every other day for 12 days in both WT and transgenic maize, when the soil water content (SWC) decreased from $40 \%$ to near $2 \%$. During the first 2 days (SWC $>30 \%$ ), the 
a

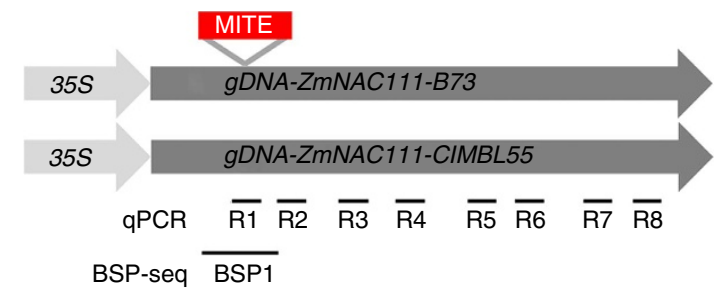

C

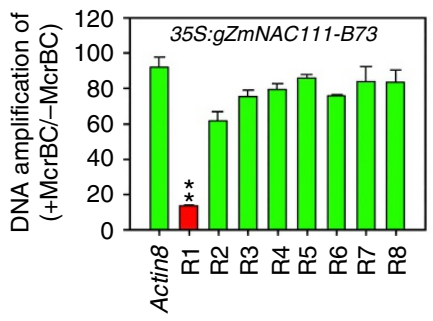

e
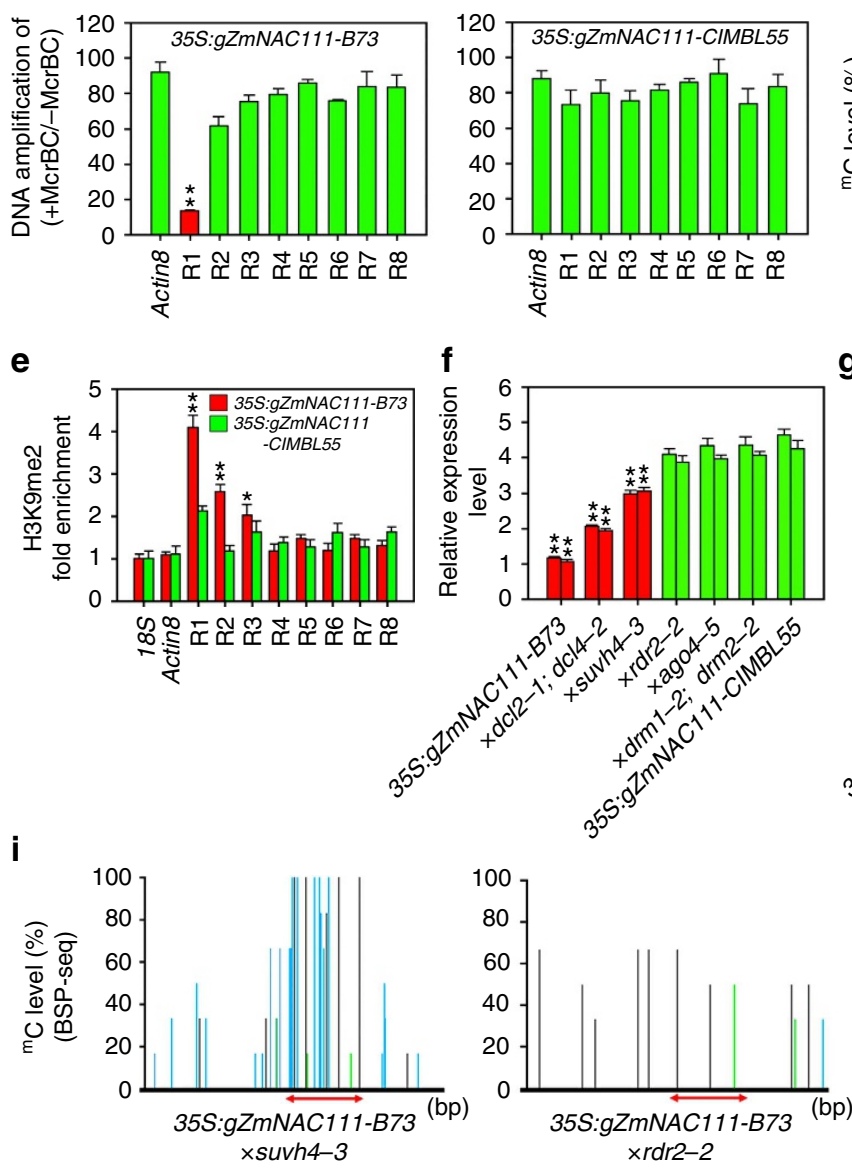

d

g
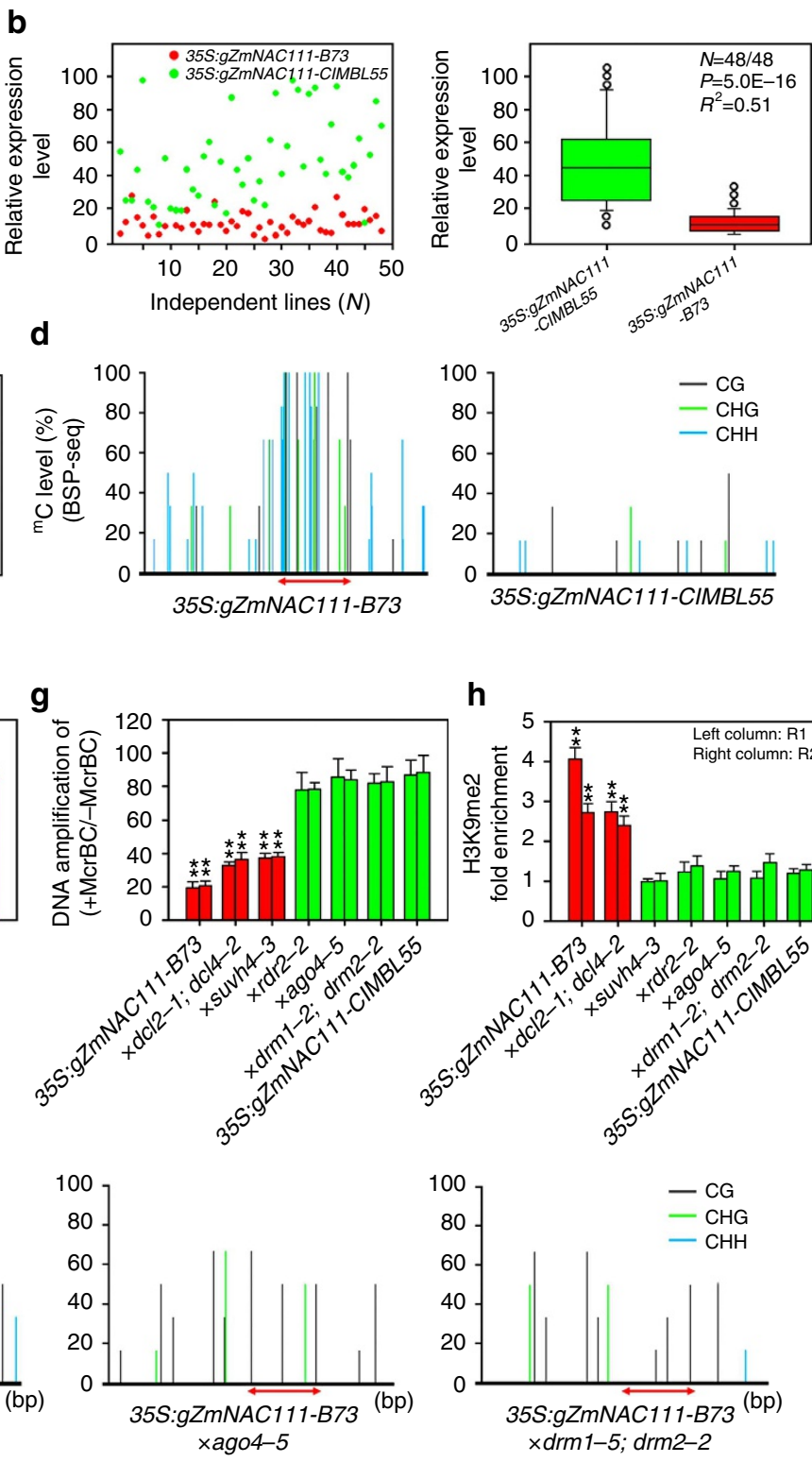

h
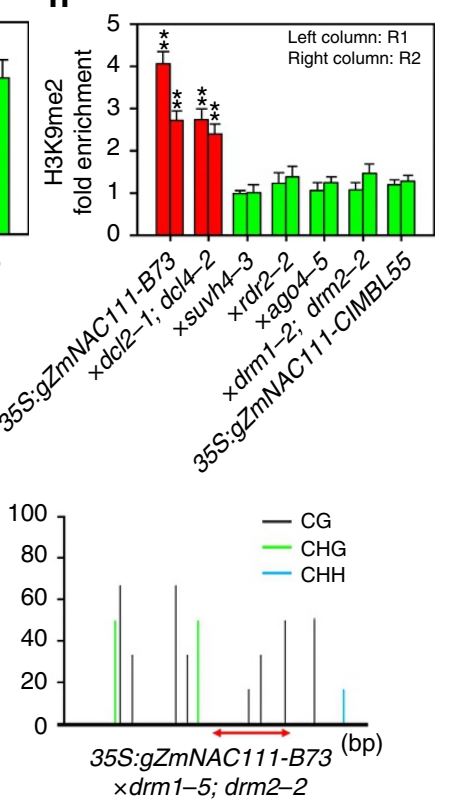

Figure 4 | Repression of ZmNAC111 expression by the MITE insertion is dependent on RNA-directed DNA methylation and histone methylation when heterologously expressed. (a) Diagram of the plasmid constructs (35S:gZmNAC111-B73 and 35S:gZmNAC111-CIMBL55) that were used to transform Arabidopsis. The MITE insertion (red box), regions of the McrBC-qPCR, ChIP-qPCR (R1-R8) and bisulphite sequencing (BSP1) analyses are indicated. (b) Left panel: comparison of ZmNAC111 expression in independent transgenic lines of 35S:gZmNAC111-B73 and 35S:gZmNAC111-CIMBL55. Right panel: statistical differences in the ZmNAC111 gene expression in the transgenic Arabidopsis lines. (c) DNA methylation status in the eight regions (R1-R8) of the genomic sequence of 35S:gZmNAC111-B73 and 35S:gZmNAC111-CIMBL55 determined by McrBC-qPCR assays. (d) Methylation states of cytosine residues in CG, CHG and CHH sites, assayed with bisulphite sequencing of the BSP1 region. (e) Chromatin states detected at the eight regions (R1-R8), using an anti-H3K9me2 ChIP-qPCR assay. The H3K9me2 states of the $18 \mathrm{~S}$ and Actin8 Arabidopsis genes were evaluated in parallel and served as negative controls. Anti-H3 was used as an internal reference for ChIP-qPCR. (f) qRT-PCR analysis of transcript levels of 35S:gZmNAC111-B73 in wild-type and the RdDM mutants, and 355:gZmNAC111-CIMBL55 transcript levels in the wild type. (g) DNA methylation status determined using the McrBC-qPCR assay of the R1 region in the designated genetic backgrounds. (h) Chromatin states detected using anti-H3K9me2 ChIP-qPCR assays at R1 (left column) and R2 (right column) region. ' $\times$ dcl2-1; dcl4-2'; ' $\times$ suvh4-3'; ' $\times$ rdr2-2'; ' $\times$ ago4-5'; and ' $\times$ drm7-2; drm2-2' in (f) and $(\mathbf{g})$ indicate the homozygous genetic background of the 35S:gZmNAC111-B73-2 (left column) and -23 (right column) transgenics after crossing. Green columns indicate that ZmNAC111 expression (f), DNA methylation (g) and H3K9me2 (h) were comparable with levels in the 35S:gZmNAC111-CIMBL55 transgenics in the wild-type background, whereas red columns indicate that they are significantly different with those in the 355:gZmNAC111-CIMBL55 transgenics. (i) Methylation of cytosine residues assayed with bisulphite sequencing of the BSP1 region of the 35S:gZmNAC111-B73 transgenics in the different RdDM-defective mutant backgrounds. The MITE region is indicated by double-sided arrows. Error bars are s.d. and significant differences are determined using the $t$-test, ${ }^{\star} P<0.05 ;{ }^{\star \star} P<0.01$.

three physiological parameters were comparable between the transgenics and WT, supporting the observation that they did not differ in growth under unstressed conditions (Fig. 5e-g). When SWC decreased to about $20 \%$, TR of the transgenics was initially measured to decrease, resulting in a significantly greater WUE (calculated as PS in relation to TR) of the transgenic maize plants relative to WT (Fig. $5 \mathrm{~g}, \mathrm{~h}$ ). With the SWC dropping to $\sim 15 \%$, the SC and TR became significantly smaller in the transgenics than 
a

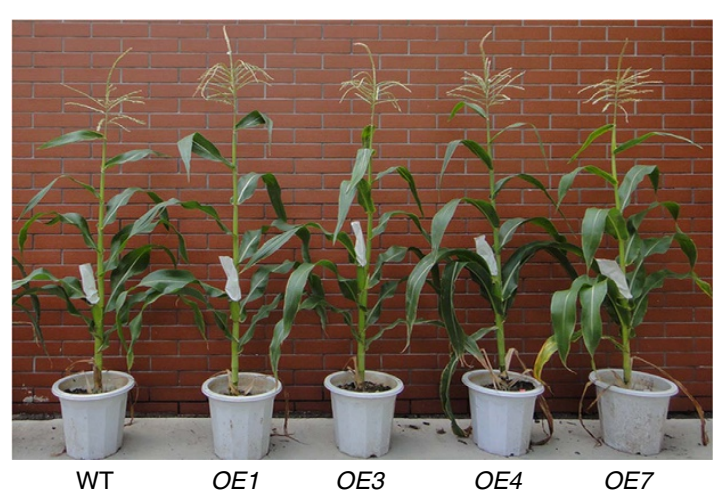

c

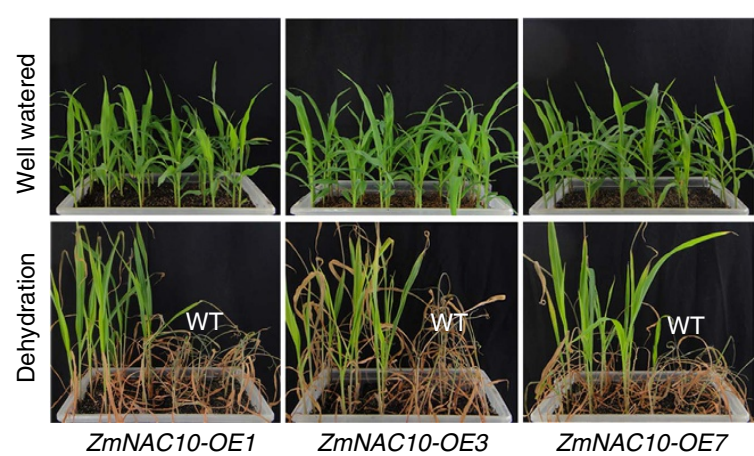

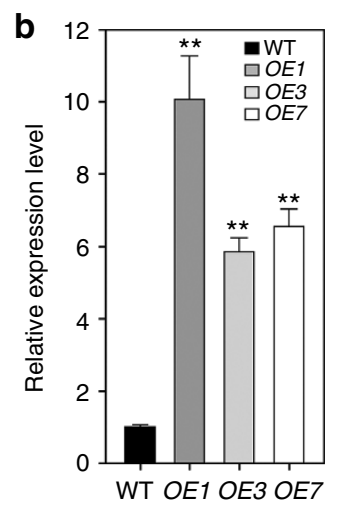

d

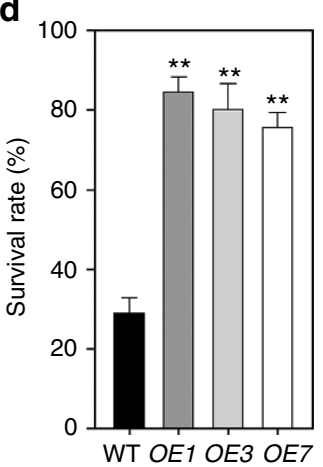

e

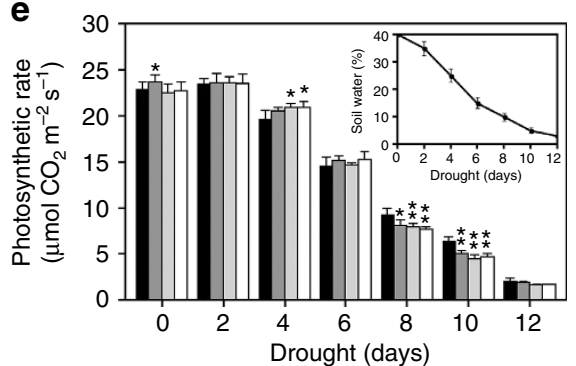

g

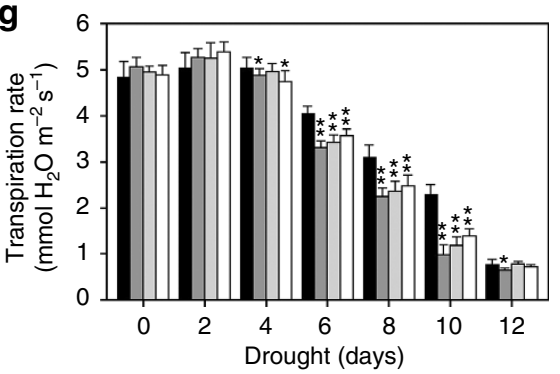

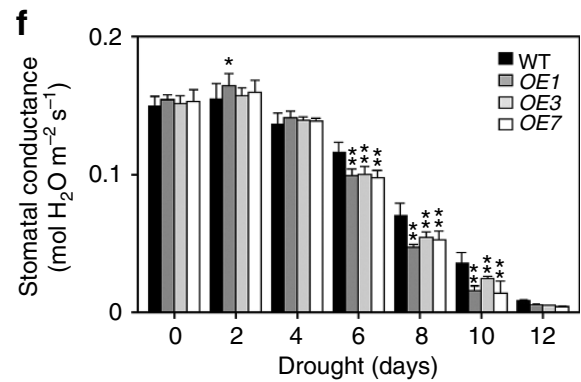

h

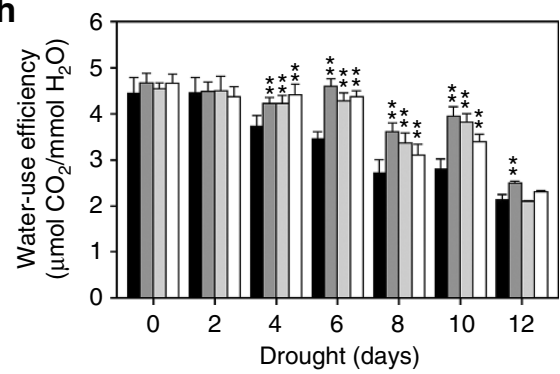

Figure 5 | Drought tolerance of ZmUbi:ZmNAC111 transgenic maize. (a) The growth phenotype of the $T_{1}$ generation transgenic and sibling transformation-negative (WT) plants. Four representative independent transgene-positive lines (ZmNAC111-OE1, OE3, OE4 and OE7) and the WT are shown. (b) Transcript levels of ZmNAC111 in the WT, and three independent ZmUbi:ZmNAC111 transgenic maize lines. (c) Drought tolerance of $T_{2}$ seedlings of ZmUbi:ZmNAC111 transgenic maize compared with WT. Photographs were taken under well-watered conditions and subsequent to a drought treatment followed by re-watering for a period of 7 days. The survival rates of WT and transgenic ZmUbi:ZmNAC111-OE1, OE3 and OE7 plants were compared. (d) Statistical analysis of survival rates after drought treatment and recovery. The average percentage of survival and standard errors were calculated from four independent experiments. (e-h) Comparison of the photosynthetic performance of ZmUbi:ZmNAC111 transgenic and WT plants during the process of the drought stress. (e) Photosynthesis rate; (f) stomatal conductance; ( $(\mathbf{g})$ transpiration rate; and (h) water-use efficiency. Error bars are s.d. and significant differences are determined using the $t$-test, ${ }^{\star} P<0.05 ;{ }^{\star \star} P<0.01$.

those in WT, whereas, the PS remained comparable (Fig. 5e). Thus, WUE of the transgenic maize was maintained greater than that of the WT (Fig. 5g). Afterwards, the PS of the transgenics was more reduced compared with that of WT; however, due to a more remarkably reduced SC and TR, the WUE of the transgenics still remained significantly greater than that of WT, until SWC dropped to $\sim 2 \%$ (Fig. $5 \mathrm{~h}$ ). These data revealed that the transgenic maize plants had a greater WUE under water deficit in comparison with WT, which likely conferred enhanced drought tolerance to the transgenic maize. 
In the next line of our study, we compared the transcriptome of the transgenic maize and WT plants under favourable and drought conditions. A total of 628 and 443 genes were found to be upregulated and downregulated by twofold in the transgenics compared with WT, respectively, under well-watered conditions (Supplementary Fig. 10; Supplementary Data 4). Under drought stress, 547 and 425 genes were twofold upregulated and downregulated in the transgenic maize relative to WT, respectively (Fig. 6a,b; Supplementary Data 5). Biological pathways responsive to abscisic acid, ethylene and abiotic stimuli were greatly enriched among these identified upregulated genes, whereas those responsive to oxidative changes and gibberellins were especially enriched among the downregulated genes (Fig. 6c). Genes responsive to abiotic and water stresses were more significantly enriched in the upregulated genes in the drought-stressed samples as compared with the untreated ones (Fig. 6c; Supplementary Fig. 10c). We hypothesize that these transcriptomic changes may contribute to the early reduction in TR, quick stomatal closure and better protection of the photosynthesis machinery of transgenic maize under drought stress. Increased expression patterns of several well-known drought-inducible genes, such as the maize homologues of NCED3 (ref. 43), AFP3 (ref. 44), RAB18 (ref. 45), RD29B (ref. 46), AHG1 (ref. 47), RD17 (ref. 45), and DREB1D (ref. 48) were verified in the transgenic maize (Fig. 6d). Most of these genes contain copies of NAC recognition core sequence $(\mathrm{CACG})^{49,50}$ in their promoters, suggesting that they might be direct target genes of ZmNAC111 (Supplementary Data 6).

Evolutionary aspects of the $Z \mathbf{m N A C 1 1 1}$ locus. Teosinte (Z. mays ssp.), a type of wild Mexican grass, is recognized as the direct progenitor of maize based on the fact that the natural cross of teosinte and maize are fertile and the availability of a wealth of genetic domestication information ${ }^{8,9}$. After domestication from teosinte, maize cultivation spread from TST to temperate regions and maize became a major crop plant providing nutritional calories for consumption by human beings. We were interested to know the presence and distribution of the 82-bp MITE insertion in the teosinte ZmNAC111 locus. When 96 teosinte accessions were genotyped, none of them were found to carry the MITE insertion at ZmNAC111 indicating that this insertion might have occurred after the domestication of maize (Supplementary Data 7). Moreover, among 116 TST inbred lines, $10.34 \%$ of them were MITE $^{+}$. Whereas, among 146 temperate lines (including stiff-stalk (SS), non-stiff-stalk (NSS) and mixed origins $^{51}$ ), $41.78 \%$ were MITE ${ }^{+}$(Fig. 6e). Nucleotide diversity at the ZmNAC111 locus was found to decrease from teosinte to TST and then to temperate maize (Fig. 6f). This finding suggested that with the spread of maize cultivation from TST to temperate regions, the $\mathrm{MITE}^{+}$genotype was accumulated especially in temperate maize germplasm. The MITE insertion in ZmNAC111 locus may compromise drought tolerance of temperate maize varieties, which is in agreement with the observation that temperate subpopulation was averagely more susceptible to drought than the TST subpopulation in the whole natural variation population. Thus, we propose that the selection of the $\mathrm{MITE}^{-}$genotype may help to improve drought tolerance in temperate maize inbred lines.

\section{Discussion}

In this study, we reported that the natural variation of $\mathrm{ZmNAC111}$ gene is associated with maize drought tolerance on the whole-genome scale. On the basis of our combined GWAS and transgenic approach, we propose that the causative variation at the ZmNAC111 locus is likely an 82-bp MITE insertion in the gene promoter, which may repress the gene expression through DNA and histone hypermethylation via the RdDM pathway. Our findings highlight the likely regulatory function of a TE in maize stress response and provide important insights into the genetic basis of the natural variation in maize drought tolerance; as well as new genetic strategies for improving this trait.

We identified the 82-bp MITE insertion in a location that was 572-bp upstream of the ZmNAC111 coding region, which was correlated with lower ZmNAC111 expression and drought susceptibility (Fig. 2b). Bisulphite-seq and ChIP-qPCR analyses revealed that DNA and histones are hypermethylated in the $Z m N A C 111$ locus in the maize inbred lines carrying the MITE (Fig. 3). The repression of ZmNAC111 expression could be reproduced when the genomic fragment containing the MITE and $Z m N A C 111$ was transferred into Arabidopsis, indicating that the underlying molecular mechanism is likely conserved across plant species. Importantly, using the Arabidopsis RdDM-defective mutants, we demonstrated that the MITE-mediated ZmNAC111 repression, at least when heterologously expressed, is dependent on RdDM (Fig. 4); which is a well-characterized RNA interference-related transcriptional gene silencing mechanism in plants $^{32}$. This modification induces and reinforces transcriptional silencing of TEs, as well as the genes that harbor or are adjacent to the TEs ${ }^{26}$. Recently, whole-genome DNA methylation and RdDM surveys in maize suggest that 24-nt siRNAs are much more highly associated with transposons, which tend to be close to genes rather than the heterochromatin regions ${ }^{52}$. Although the majority of the maize genome exists in a heterochromatic status, which is marked by $\mathrm{H} 3 \mathrm{~K} 9 \mathrm{me} 2$ and $\mathrm{H} 3 \mathrm{~K} 27 \mathrm{me} 2$, RdDM was only observed to be near gene-coding regions ${ }^{53}$. As a result, it gave rise to the formation of $\mathrm{CHH}$ islands predominantly near genes, rather than in the repetitive intergenic DNA regions ${ }^{52,53}$. Our findings provide a potential molecular mechanism for how the 82-bp MITE interplays with its adjacent gene so as to contribute to drought tolerance variance in the natural maize population.

TFs play important roles in the regulation of gene expression in response to abiotic stresses, and their molecular engineering is proposed as a potential strategy for the genetic improvement of stress tolerance in crops 54,55 . NAC proteins constitute a plantspecific superfamily whose members participate in various regulatory and developmental processes, including stress response and tolerance ${ }^{42}$. The typical NAC proteins share a conserved N-terminal DNA domain but vary greatly in other regions, resulting in distinct functions of different proteins. In maize, at least 116 predicted NAC members have been identified $^{35}$. Although $\mathrm{ZmNAC111}$ was classified into an identical phylogenetic clade with OsNAC10 among the annotated NAC proteins examined (Supplementary Fig. 2), ZmNAC111 and OsNAC10 only share a $48 \%$ sequence identity on the full protein level indicating both sequence similarity and diversity between them. Previous reverse genetic studies reported that increasing OsNAC10 expression in roots improved the yield of transgenic rice under drought ${ }^{36}$. Expression analysis of $Z m N A C 111$ in leaf samples of 133 natural maize varieties indicated that $Z m N A C 111$ expression was positively correlated with seedling drought tolerance under moderate and severe stresses (Fig. 2). These data suggested a positive regulatory role of $Z m N A C 111$ in maize seedlings exposed to water stress. Moreover, transgenic studies in both Arabidopsis and maize demonstrated that overexpression of the ZmNAC111 gene could improve drought tolerance of transgenic plants and modulated stomatal closure and droughtresponsive gene expression. These observations support our proposal that the elevated expression of $Z m N A C 111$ gene contributes to maize drought tolerance and that $Z m N A C 111$ acts as a positive regulator of drought response in maize (Fig. 5; Supplementary Fig. 9). Comparative transcriptome analysis of the 
a Up regulated genes

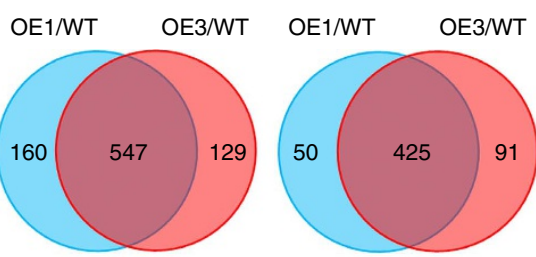

b

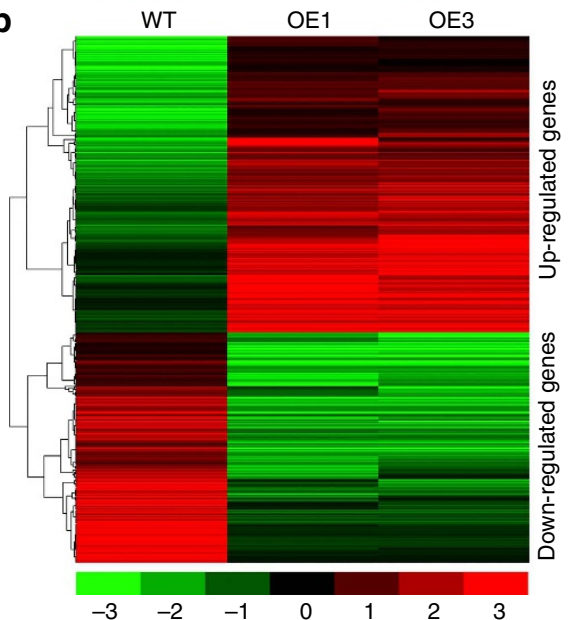

C

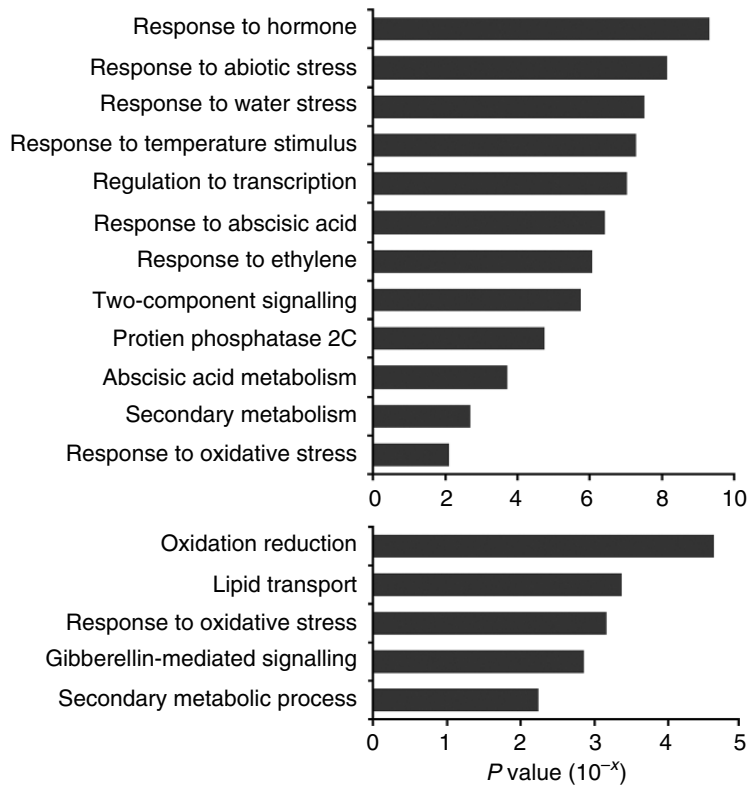

d
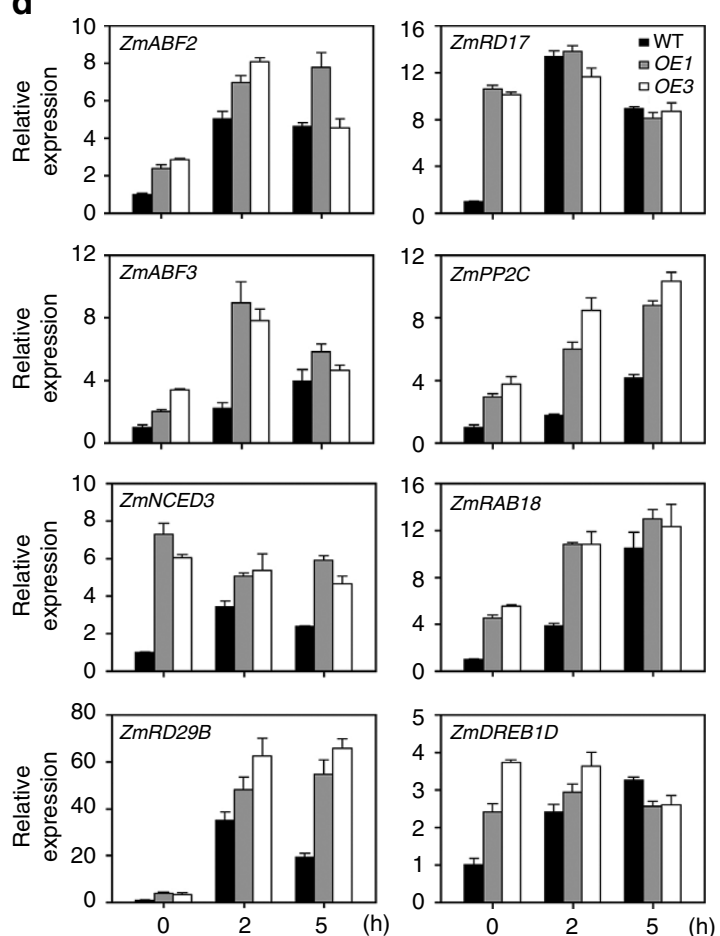

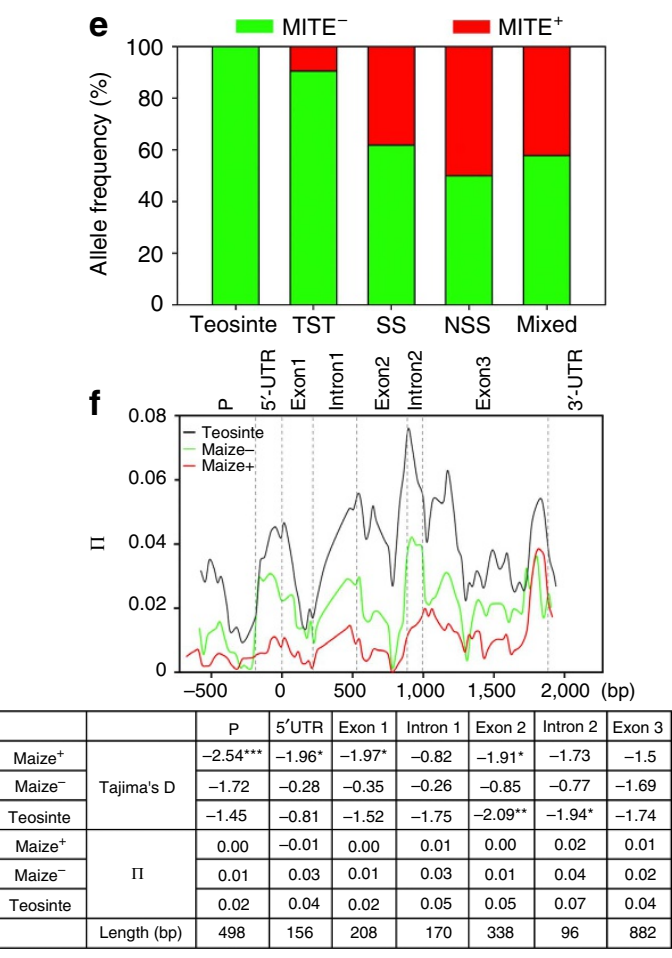

Figure 6 | Transcriptomic analysis of ZmUbi:ZmNAC111 transgenic maize and comparison of MITE insertion frequency and nucleotide diversity of ZmNAC111 in teosinte, TST and temperate maize inbred lines. (a) Venn diagrams of upregulated or downregulated genes in ZmUbi:ZmNAC111-OE1 and OE3 plants relative to WT plants using a significance cutoff of $P<0.001$, and a fold change $(F C)>2$. (b) Hierarchical clustering of differentially expressed genes in the transgenic lines relative to WT plants. The indicated scale is the $\log _{2}$ value of the normalized level of gene expression. (c) Gene ontology of biological pathways enriched in the transgenic lines based on the upregulated or downregulated genes. (d) qRT-PCR verification of increased expression of genes involved in plant drought response and tolerance in the transgenics under normal and drought conditions. Error bars are s.d. (e) Frequency of MITE insertion in the ZmNAC111 promoter in teosinte, TST and temperate (including non-stiff stalk (NSS), stiff stalk (SS) and mixed) ${ }^{51}$ maize inbred lines. (f) Nucleotide diversity at the ZmNAC111 locus among teosinte and $\mathrm{MITE}^{-}$(maize ${ }^{-}$) and $\mathrm{MITE}^{+}$(maize ${ }^{+}$) inbred lines of maize. Nucleotide diversity was compared across the ZmNAC111 locus, among the $72 \mathrm{MITE}^{+}$and $190 \mathrm{MITE}^{-}$maize inbred lines and 42 teosinte entries. ' $\mathrm{P}^{\prime}$ denotes the $Z \mathrm{mNAC111}$ promoter region. Nucleotide diversity $(\pi)$ for teosinte $\left(\pi_{\mathrm{T}}\right.$, grey), the maize inbred lines of maize ${ }^{+}\left(\pi_{+}\right.$, red) and Maize ${ }^{-}\left(\pi_{-}\right.$, green) was calculated using a 100-bp sliding window and 25-bp step. The Tajima's $D$ values of different regions are shown. ${ }^{\star} P<0.05 ;{ }^{\star \star} P<0.01 ;{ }^{\star \star \star} P<0.001$ 
transgenic maize and WT determined that a number of genes involved in ABA-responsiveness, such as $Z m N C E D 3, Z m R A B 18$, $Z m R D 29 B, Z m R D 17$ and $Z m P P 2 C$, were upregulated indicating that $Z m N A C 111$ might function in an ABA-dependent stressresponsive pathway (Fig. 6d). In agreement with this result, Arabidopsis transgenic plants were more sensitive to exogenous ABA treatment in regard to germination and stomatal closure (Supplementary Fig. 9). In transgenic maize, the leaf SC and TR were more responsive to water deficit during the decrease of SWC, from 15 to $\sim 2 \%$, in the drought treatment (Fig. $5 \mathrm{e}-\mathrm{h}$ ). On the basis of these results, we suggest that $\mathrm{ABA}$-dependent regulation was enhanced in the $Z m N A C 111$ transgenic plants.

It is considered that enhancing effective use of water, which implies maximal soil moisture capture reduced non-stomatal water loss and management for minimal soil evaporation, is important for drought tolerance improvement under field conditions; however, improving WUE by reducing SC and TR may diminish plant yield in fields ${ }^{56}$. In this research, we did not observe obvious morphological changes in transgenic plant overexpressing ZmNAC111 in comparison with the control plants, when they grew in pots under favourable greenhouse conditions (Fig. 5a). This was supported by the comparable measurements of the leaf PS between the transgenic and WT plants (Fig. 5e). Only on drought stress, a greater WUE was revealed in transgenic maize in comparison with WT (Fig. 5h). A similar phenomenon was also observed in transgenic rice overexpressing SNAC1 (ref. 50). The early reduction of TR was observed in transgenic maize, when the SC remained comparable between the transgenic maize and WT (SWC>20\%); which was probably due to a better or quicker osmo-adjustment (Fig. 5g). In spite of the more highly reduced SC and TR in the transgenic maize as compared with WT, the leaf PS was hardly affected when SWC was $\sim 15 \%$ (Fig. 5e,f). These results indicated a better protection of photosynthesis machinery or maintenance of cellular oxidative status under drought stress in the transgenic maize. Thus, the improved drought tolerance and greater WUE of transgenic maize were likely attributed to enhanced $\mathrm{ABA}$ signalling, quicker osmo-adjustment, better cellular protection in response to drought stress, rather than the consequence of plant growth retention. These findings also suggest that efficient water usage of plants can be improved both physiologically and genetically. Nevertheless, further intensive evaluations on important agronomic traits of transgenic plants under field conditions are needed with regard to the concerns of gene application to maize production.

In addition to SNPs, TE presence/absence variations are common and widely distributed in the maize genome, which is considered as driving force for crop evolution and domestication $^{57}$. The MITE insertion was only present in maize germplasm but not in the teosinte accessions we examined (Fig. 6e). This finding suggests that the MITE may have inserted into $Z m N A C 111$ locus after maize domestication from its wild ancestor. The domestication of crops from their wild ancestors may cause the loss of genes or alleles, which are responsible for tolerance to various environmental stresses. Recently, it has been reported that the deletion of the $Z m W A K$ gene during maize domestication increases susceptibility of domesticated cultivars to head smut, which is a major disease in maize production ${ }^{12}$. Decrease in plant stress tolerance might be exaggerated if the stress pressure is not present during the selection in breeding programme, in which high yield but not stress tolerance, is the primary goal. No evident adverse effect was observed on plant normal growth and development of the $Z m N A C 111$ transgenic Arabidopsis and maize under the favourable conditions. However, further investigation is required to test if enhancement of the $Z m N A C 111$ expression results in additional undesired phenotypes in field conditions. In addition, the ZmNAC111 locus was not found to be associated with 17 important agronomic traits by analysing 513 maize inbred lines ${ }^{58}$. Therefore, $Z m N A C 111$ may be a potential candidate in gene engineering, and its $\mathrm{MITE}^{-}$allele could be a selection target for the genetic improvement of drought tolerance in maize. It should be noticed that in the present study, maize drought tolerance was evaluated at seedling stage in pot-cultivated plants, which limited the characterization of above-ground tissues and vegetative growth. Whether ZmNAC111 and its $\mathrm{MITE}^{-}$allele can significantly contribute to maize yield under drought in fields demands further field-based investigation. In addition, the MITE insertion seems to be especially common in temperate maize germplasm and whether this allele confers any advantage in breeding programmes aimed for temperate regions may be an interesting theme for future research.

\section{Methods}

ZmNAC111-gene association mapping. GWAS of maize drought-tolerant genes was performed by analysing a maize natural variation panel consisting of 368 inbred lines collected from TST and temperate regions ${ }^{19}$. Plant drought tolerance of different inbred lines was phenotypied as previously described ${ }^{24}$. Briefly, the natural variation panel of maize consisting of 368 maize inbred lines was planted in a cultivation pool $(6 \times 1.4 \times 0.22 \mathrm{~m}$, length $\times$ width $\times$ depth $)$ in which 5 ton of loam were mixed with 0.25 ton of chicken manure. To phenotype the drought tolerance of each genotype, watering was withheld when the seedlings developed three true leaves. Re-watering was applied to recover the surviving plants when clear wilting difference was observed. After rehydration for 6 days, the SR of each genotype was scored. The phenotypic data were obtained from six replicated experiments. The 56,110 genomic and 525,105 transcriptomic SNPs, with minor allele frequency (MAF) $\geq 0.05$ were used for GWAS. The standard mixed linear model was applied (TASSEL 3.1.0) $)^{59}$, in which the population structure (Q) and kinship (K) were estimated as previously described ${ }^{24}$. Briefly, principal components of the association panel were calculated by EIGENSTRAT ${ }^{60}$ using the high-quality 525,105 SNP data with MAF $\geq 0.05$ (ref. 61). The first two dimensions were used in the principal component analysis to estimate the population structure, which could explain the $11.01 \%$ of the phenotypic variation. These results were comparable to those that were calculated by STRUCTURE. The analysis was completed by the $\mathrm{lm}$ function in an R program. Single-marker association analysis was initially performed to filter out markers that had no relationship with the trait $(P \geq 0.995)$ Subsequently, 1,822 SNP markers on each chromosome were chosen to estimate the kinship coefficient $(\mathrm{K})$ by SPAGeDi. Markers that were in approximate linkage equilibrium with each other were determined from PLINK ${ }^{62}$ based on SNP pruning (window size 50, step size 50 and the LD $R^{2}$ threshold is 0.2 ), and the number of the subset markers was 85,806 . The suggestive $P$ value threshold to control the genome-wide type 1 error rate was $1.17 \times 10^{-5}$, which was considered as the significance cutoff for the association. ZmNAC111-based association mapping was performed within 146 temperate and 116 TST maize inbred lines, which were representative of the whole population. The $Z m N A C 111$ promoter $(\sim 0.7 \mathrm{~kb})$, coding regions (including introns) and $5^{\prime}-$ and $3^{\prime}$-UTR sequences were amplified and sequenced. These sequences were assembled using ContigExpress in Vector NTI Advance 10 (Invitrogen) and aligned using MEGA version 5 (http://megasoftware.net/). Polymorphisms (SNPs and InDels) were identified and their association to drought tolerance was calculated again by TASSEL 3.1.0, under the standard MLM, with MAF $\geq 0.05$.

ZmNAC111 gene expression analysis in different inbred lines. ZmNAC111 expression was analysed in 133 maize inbred lines. Drought treatment was applied to the soil-grown plants at the three-leaf seedling stage by withholding water. Leaf samples were collected when the relative leaf water content (RLWC) decreased from $\sim 98-70 \%$, and to $58 \%$. Total RNA of 399 samples was isolated using TRIzol reagent (Biotopped) from a minimum of three seedlings. RNA was treated with RNase-free DNase I (Takara), and single-stranded cDNA was synthesized using recombinant M-MLV reverse transcriptase (Promega). The quantification method $\left(2^{-\Delta \mathrm{Ct}}\right)^{63}$ was used and the variation in expression was estimated using three biological replicates. The maize Ubi-2 (UniProtKB/TrEMBL; ACC: Q42415) gene was used as an internal control to normalize the data. PCR conditions consisted of an initial denaturation step at $95^{\circ} \mathrm{C}$ for $10 \mathrm{~min}$, followed by 40 cycles at $95^{\circ} \mathrm{C}$ for $15 \mathrm{~s}, 60^{\circ} \mathrm{C}$ for $30 \mathrm{~s}$.

Allelic effect of ZmNAC111 in maize segregating populations. Three $\mathrm{F}_{2: 3}$ segregating populations (CIMBL91 $\times$ BY4944, CIMBL55 $\times$ GEMS54 and CIMBL55 $\times$ CIMBL9) were constructed. The genotype at the $Z m N A C 111$ locus was analysed in $\sim 200$ individual $F_{2}$ plants in each population. Polymorphisms in the PCR products were visualized on $2 \%$ agarose gels. Homozygous $F_{2}$ individuals 
at the ZmNAC111 locus of the tolerant allele (MITE ${ }^{-}$) and the sensitive allele $\left(\mathrm{MITE}^{+}\right.$) were self-pollinated to obtain $\mathrm{F}_{3}$ progenies. $\mathrm{F}_{3}$ progenies that were homozygous at the InDel-572 locus (MITE ${ }^{-/-}$or MITE $^{+/+}$) were mixed, respectively. Two types of $\mathrm{F}_{3}$ plants were grown in enriched soil (soil to vermiculite in a ratio of 1:1) in plastic boxes $(0.70 \times 0.50 \times 0.18 \mathrm{~m}$, length $\times$ width $\times$ depth $)$ and their drought tolerance was evaluated. Each box contained 90 seedlings for each type of $\mathrm{F}_{3}$ plants. Three independent replications were performed in a greenhouse using 16 -h light $/ 8$-h dark, $28 / 22^{\circ} \mathrm{C}$ and a room humidity of $60 \%$ to obtain the statistical data. Drought was applied to the 10-day-old plants by withholding water. When SWC decreased from $40 \%$ to near $0 \%$, and wilting and death of the seedlings were visible, plants were re-watered to identify surviving plants. The SR of each genotype was recorded. Three replications were carried out for statistical analyses.

McrBC-based DNA methylation assay. Genomic DNA was isolated from fresh young leaves collected from B73 and CIMBL55 maize lines before $($ RLWC $=98 \%)$ or after drought treatment (RLWC $=70 \%)$. DNA $(1 \mu \mathrm{g})$ was digested for $16 \mathrm{~h}$ at $37^{\circ} \mathrm{C}$ with 10 units of McrBC enzyme, a DNA methylation-sensitive enzyme (Takara), in parallel with a mock reaction; $50 \mathrm{ng}$ of digested DNA was used for qPCR reactions. DNA hypermethylation was demonstrated by the lower amount of amplification products in the $\mathrm{qPCR}$ analysis. All results were obtained by digesting at least two biological replicates and two independent McrBC digests. qPCR was performed using the following conditions: step $1: 95^{\circ} \mathrm{C}, 10 \mathrm{~min}$; step 2: $95^{\circ} \mathrm{C}, 15 \mathrm{~s}$; $60^{\circ} \mathrm{C}, 30 \mathrm{~s}$ (40 cycles). McrBC digestion at the $Z m N A C 111$ gene was normalized to the reference gene maize Ubi-2 and Actin1 and then to the undigested control. Arabidopsis plants were grown on Murashige and Skoog (MS) medium agar plates for 21 days before collection. Actin 8 was used as the reference gene in Arabidopsis ${ }^{64}$. Digestion levels have been inverted to represent methylation levels.

Bisulphite analysis. Bisulphite treatment was performed on $200 \mathrm{ng}$ of genomic DNA by using the EZ DNA Methylation-Gold kit (Zymo Research, Orange, CA). After bisulphite conversion, the treated DNA was amplified by PCR. Amplified fragments were cloned into the pGEM-T vector (Promega) for sequencing. At least eight clones of each genotype were sequenced.

ChIP assay. Fresh young leaves were collected from B73 and CIMBL55 maize lines grown under normal and drought conditions as described above. Whole plants of Arabidopsis were collected from 35S:ZmNAC111-B73 and 35S:ZmNAC111CIMBL55 lines grown on the MS agar plates for 21 days. ChIP assays were performed as previously described ${ }^{65}$. Approximately, $7 \mu \mathrm{l}$ of anti-H3 (Abcam; ab1791) or anti-H3K9me2 (Abcam; ab1220) antibodies were used for the ChIP assays. The amount of immunoprecipitated ZmNAC111 chromatin was determined by qPCR on different regions of the ZmNAC111 locus. Maize Ubi-2 and Actin8 were used as internal controls for maize and Arabidopsis, respectively. The relative abundance was normalized to the amount of DNA immunoprecipitated by a Histone 3-specific antibody.

Generation of transgenics in RdDM mutant backgrounds. Two 35S:gZmNAC111B73-2, -23 and 35S:gZmNAC111-CIMBL55-5, -12 homozygous $\mathrm{T}_{2}$ lines were crossed with five mutants: dcl2-1;dcl3-1;dcl4-2 (CS16391), drm1-2;drm2-2 (CS16383), ago4-5 (CS9927), rdr2-2 (SALK_059661) and suvh4-3 (Salk_105816) (http:// www.arabidopsis.org). Through PCR-based genotyping analysis, at least three independent $\mathrm{F}_{2}$ homozygous plants for each cross were obtained and collected. Since the DCL3 locus was heterozygous, only 35S:gZmNAC111-B73-2 and -23 in the dcl2-1;dcl4-2 mutant background were obtained. The ChIP assay was performed using $\mathrm{F}_{3} \mathrm{RdDM}$-mutant homozygous plants obtained from crossing the two independent transgenic Arabidopsis with the RdDM mutants. The $\mathrm{F}_{3}$ plants germinated on kanamycin-selective medium were used for further DNA methylation and ChIP analyses. Arabidopsis T-DNA insertion lines were obtained from the Arabidopsis Biological Resource Center.

Arabidopsis drought tolerance assays. The $Z m N A C 111$ genomic region in the B73 and CIMBL55 inbred lines and, the coding region in B73 were amplified and inserted into the pGreen vector ${ }^{66}$ under the CaMV 355 promoter using the NotI and XhoI restriction sites. The constructed plasmid was transformed into the GV3101 Agrobacterium tumefaciens strain containing the pSoup helper plasmid. Arabidopsis thaliana ecotype Col-0 was transformed by Agrobacterium-mediated transformation and independent $\mathrm{T}_{2}$ transgenic lines were obtained using kanamycin-based selection. ZmNAC111 gene expression in transgenics was determined by qRT-PCR, in which Actin8 was used as an internal control for normalization. For the drought tolerance assays, 7 -day-old plants were transferred into pots containing $250 \mathrm{~g}$ of soil. Thirty-two-day-old plants growing under favourable water conditions were exposed to drought stress. Water was withheld from the plants for 14 days. Watering was then resumed to allow the plants to recover. Six days later, the number of surviving plants was recorded. At least 64 plants of each line were compared with empty-vector transformed (VC) plants in each test, and statistical data were based on data obtained from three independent experiments.
ABA-sensitivity in the transgenic Arabidopsis. The VC and 35S:ZmNAC111 transgenic plants were grown in parallel and collected. Seeds obtained from these plants were planted on $1 / 2 \times$ MS plates containing $1 \%$ sucrose and were supplemented with or without different concentrations of ABA. Plates were chilled at $4{ }^{\circ} \mathrm{C}$ in the dark for 3 days for stratification and moved to $22^{\circ} \mathrm{C}$ with a 16 -h light/8-h dark cycle. Germination (emergence of radicals) was scored on the third day after germination, with three replicated assays. Stomatal aperture assays were conducted as previously described ${ }^{67}$. Briefly, rosette leaf peels were floated in a stomatal opening solution (10 mM MES-Tris (pH 6.15), $100 \mu \mathrm{M} \mathrm{CaCl}_{2}$ and $10 \mathrm{mM}$ $\mathrm{KCl}$ ) for $2 \mathrm{~h}$ and then transferred to a solution supplemented with various concentrations of $\mathrm{ABA}(0,0.1,1$ and $10 \mu \mathrm{M})$ for another $2 \mathrm{~h}$. Subsequently, the abaxial surface of each leaf was applied to 3-M clear tape to peel off the epidermal layer. Stomatal apertures were imaged and measured using ImageJ software. Forty-five stomatal apertures were analysed in each experiment and the reported values represent the mean \pm s.d.

Generation and analysis of the transgenic maize. The coding region of $Z m N A C 111$ was amplified from B73 and the sequence-confirmed PCR fragment was inserted into the pSBII vector under the control of the Zmubil promoter. The pSBI plasmid was then inserted into the LBA4404 A. tumefaciens strain. The LBA4404 strain, with the integrated pSBIII plasmid, was then used to deliver the Zmubil:ZmNAC111 expression cassette into the A188 maize inbred line as described $^{68}$. Transgenic $\mathrm{T}_{0}, \mathrm{~T}_{1}$ and $\mathrm{T}_{2}$ plants were grown in a greenhouse under a 16-h-light/8-h-dark condition. Transgenic-positive and the sibling transgenicnegative (WT) plants were determined in each generation by PCR analysis for the transgene. The expression of $Z m N A C 111$ in transgenic plants was determined by qRT-PCR. Three independent $\mathrm{T}_{2}$ lines, ZmNAC111-OE1, ZmNAC111-OE3 and ZmNAC111-OE7, were selected for further analyses. Transgenic positive (individually genotyped by transgene-based PCR analysis) and WT plants were planted side by side in enriched soil (soil and vermiculite in a ratio of 1:1). Drought treatment was applied to the soil-grown plants at the three-leaf seedling stage by withholding water. After $\sim 20$ days, watering was resumed to allow plants to recover. The number of surviving plants was recorded 7 days later. At least 15 plants of each line were compared in each test and statistical analyses were based on data obtained from three independent experiments. SC, PS and TR of the $\mathrm{T}_{2}$ transgenic and WT plants were measured on fully expanded leaves of seedlings at the three-leaf stage using a LICOR-6400 $\mathrm{CO}_{2}$ gas exchange analyser (LICOR-6400, Lincoln, NE). SWC was recorded every other day after the initiation of water withholding. Statistical analysis was based on data obtained from seven seedlings for each plant line and the experiment was repeated twice.

RNA-seq analysis of transgenic maize. For maize RNA-seq analysis, pooled tissues from three 8-day-old maize seedlings were collected from transgenic and WT plants before or after 2-h dehydration on a clean bench to conduct the RNA-seq analysis. Total RNA was isolated using TRIzol reagent (Biotopped) and RNA integrity was evaluated using a Bioanalyzer 2100 (Agilent). The 100-bp paired-end Illumina sequencing was conducted at Berry Genomics (Beijing). An average of 3 gigabases of raw data were generated for each sample. Differential gene expression was determined using Strand NGS 2.0 software. A total of 31,501 genes were identified, representing $\sim 79 \%$ of all the predicted genes in maize. Enrichment analysis of gene ontology of biological pathways (GOBPs) was performed using the DAVID software program ${ }^{69}$ (http://david.abcc.ncifcrf.gov/) to compute $P$ values that indicate the significance of each GOBP being represented by the genes. GOBPs with $P<0.01$ were identified as enriched processes. qRT-PCR of selected genes that were determined to be critical to drought tolerance was performed to verify the RNA-seq data.

Nucleotide diversity and tests for neutrality. The genomic region of $Z m N A C 111$ was amplified and sequenced in 42 teosinte accessions. Nucleotide diversity $(\pi)$ and the Tajima's D-statistic were calculated using DnaSP version 5.0 (ref. 70).

Phylogenetic tree construction. The full-length amino acid sequences of $55 \mathrm{NAC}$ TF encoding genes identified in maize, rice, Arabidopsis and sorghum were aligned using the ClustalX 1.83 program with default parameters. The phylogenetic tree was constructed based on this alignment result using the neighbour-joining method in MEGA version 5 with the following parameters: Poisson correction, pairwise deletion, uniform rates and bootstrap (1,000 replicates).

Transactivation activity assay. cDNAs of $Z m N A C 111$ from 10 maize inbred lines were individually cloned into the pGBKT7 vector for evaluating protein transactivation activity in the AH109 yeast strain. The cell concentration of yeast transformants was adjusted to an $\mathrm{OD}_{600}$ of 0.1 , and then plated on various selective plates, $\mathrm{SD} / \mathrm{-T}, \mathrm{SD} / \mathrm{-T}-\mathrm{H}$ and $\mathrm{SD} / \mathrm{-T}-\mathrm{H}-\mathrm{A}$, to compare their survival. Plates were incubated at $30^{\circ} \mathrm{C}$ for $2-5$ days before photographing. All the primers used in this research are listed in Supplementary Data 8. 


\section{References}

1. Lobell, D. B. et al. Greater sensitivity to drought accompanies maize yield increase in the U.S. Midwest. Science 344, 516-519 (2014).

2. Pennisi, E. Plant genetics. The blue revolution, drop by drop, gene by gene. Science 320, 171-173 (2008).

3. Duvick, D. N. The contribution of breeding to yield advances, in maize (Zea mays L.). Adv. Agronomy 86, 83-145 (2005)

4. Hao, Z. et al. Meta-analysis of constitutive and adaptive QTL for drought tolerance in maize. Euphytica 174, 165-177 (2010).

5. Semagn, K. et al. Meta-analyses of QTL for grain yield and anthesis silking interval in 18 maize populations evaluated under water-stressed and well-watered environments. BMC Genomics 10, 313-328 (2013).

6. Messmer, R. et al. Drought stress and tropical maize: QTL-by-environment interactions and stability of QTLs across environments for yield components and secondary traits. Theor. Appl. Genet. 119, 913-930 (2009).

7. Almeida, G. D. et al. QTL mapping in three tropical maize populations reveals a set of constitutive and adaptive genomic regions for drought tolerance. Theor. Appl. Genet. 126, 583-600 (2013).

8. Wang, H. et al. The origin of the naked grains of maize. Nature 436, 714-719 (2005)

9. Studer, A., Zhao, Q., Ross-Ibarra, J. \& Doebley, J. Identification of a functional transposon insertion in the maize domestication gene tb1. Nat. Genet. 43, 1160-1163 (2011)

10. Salvi, S. et al. Conserved noncoding genomic sequences associated with a flowering time quantitative trait locus in maize. Proc. Natl Acad. Sci. USA 104, 11376-11381 (2007)

11. Hung, H. Y. et al. ZmCCT and the genetic basis of day-length adaptation underlying the postdomestication spread of maize. Proc. Natl Acad. Sci. USA 109, 1913-1921 (2012).

12. Zuo, W. et al. A maize wall-associated kinase confers quantitative resistance to head smut. Nat. Genet. 47, 151-157 (2015).

13. Price, A. H. Believe it or not, QTLs are accurate! Trends Plant Sci. 11, 213-216 (2006).

14. Yu, J. \& Buckler, E. S. Genetic association mapping and genome organization of maize. Curr. Opin. Biotechnol. 17, 155-160 (2006).

15. Atwell, S. et al. Genome-wide association study of 107 phenotypes in Arabidopsis thaliana inbred lines. Nature 465, 627-631 (2010).

16. Riedelsheimer, C. et al. Genome-wide association mapping of leaf metabolic profiles for dissecting complex traits in maize. Proc. Natl Acad. Sci. USA 109, 8872-8877 (2012).

17. Huang, X. et al. Genome-wide association study of flowering time and grain yield traits in a worldwide collection of rice germplasm. Nat. Genet. 44, 32-39 (2011).

18. Yan, J. et al. Rare genetic variation at Zea mays crtRB1 increases beta-carotene in maize grain. Nat. Genet. 42, 322-327 (2010).

19. Li, H. et al. Genome-wide association study dissects the genetic architecture of oil biosynthesis in maize kernels. Nat. Genet. 45, 43-50 (2013).

20. Yang, Q. et al. CACTA-like transposable element in ZmCCT attenuated photoperiod sensitivity and accelerated the postdomestication spread of maize. Proc. Natl Acad. Sci. USA 110, 16969-16974 (2013)

21. Xue, Y. et al. Genome-wide association analysis for nine agronomic traits in maize under well-watered and water-stressed conditions. Theor. Appl. Genet. 126, 2587-2596 (2013).

22. Lu, Y. et al. Joint linkage-linkage disequilibrium mapping is a powerful approach to detecting quantitative trait loci underlying drought tolerance in maize. Proc. Natl Acad. Sci. USA 107, 19585-19590 (2010).

23. Setter, T. L. et al. Genetic association mapping identifies single nucleotide polymorphisms in genes that affect abscisic acid levels in maize floral tissues during drought. J. Exp. Bot. 62, 701-716 (2011).

24. Liu, S. et al. Genome-wide analysis of $Z m D R E B$ genes and their association with natural variation in drought tolerance at seedling stage of Zea mays L. PLoS Genet. 9, e1003790 (2013).

25. Schnable, P. S. et al. The B73 maize genome: complexity, diversity, and dynamics. Science 326, 1112-1115 (2009).

26. Lisch, D. Regulation of transposable elements in maize. Curr. Opin. Plant Biol. 15, 511-516 (2012)

27. Feschotte, C., Jiang, N. \& Wessler, S. R. Plant transposable elements: where genetics meets genomics. Nat. Rev. Genet. 3, 329-341 (2002).

28. Makarevitch, I. et al. Transposable elements contribute to activation of maize genes in response to abiotic stress. PLoS Genet. 11, e1004915 (2014)

29. Castelletti, S., Tuberosa, R., Pindo, M. \& Salvi, S. A MITE transposon insertion is associated with differential methylation at the maize flowering time QTL Vgt1. G3 4, 805-812 (2014).

30. Wei, L. et al. Dicer-like 3 produces transposable element-associated 24-nt siRNAs that control agricultural traits in rice. Proc. Natl Acad. Sci. USA 111, 3877-3882 (2014)

31. Law, J. A. \& Jacobsen, S. E. Establishing, maintaining and modifying DNA methylation patterns in plants and animals. Nat. Rev. Genet. 11, 204-220 (2010).
32. Zhang, H. \& Zhu, J. K. RNA-directed DNA methylation. Curr. Opin. Plant Biol. 14, 142-147 (2011)

33. Stroud, H., Greenberg, M. V., Feng, S., Bernatavichute, Y. V. \& Jacobsen, S. E. Comprehensive analysis of silencing mutants reveals complex regulation of the Arabidopsis methylome. Cell 152, 352-364 (2013).

34. Jackson, J. P., Lindroth, A. M., Cao, X. \& Jacobsen, S. E. Control of CpNpG DNA methylation by the KRYPTONITE histone $\mathrm{H} 3$ methyltransferase. Nature 416, 556-560 (2002).

35. Voitsik, A. M., Muench, S., Deising, H. B. \& Voll, L. M. Two recently duplicated maize NAC transcription factor paralogs are induced in response to Colletotrichum graminicola infection. BMC Plant Biol. 13, 85-100 (2013)

36. Jeong, J. S. et al. Root-specific expression of OsNAC10 improves drought tolerance and grain yield in rice under field drought conditions. Plant Physiol. 153, 185-197 (2010).

37. Taoka, K. et al. The NAC domain mediates functional specificity of CUPSHAPED COTYLEDON proteins. Plant J. 40, 462-473 (2004).

38. Xie, Q., Frugis, G., Colgan, D. \& Chua, N. H. Arabidopsis NAC1 transduces auxin signal downstream of TIR1 to promote lateral root development. Genes Dev. 14, 3024-3036 (2000).

39. Mitsuda, N. et al. NAC transcription factors, NST1 and NST3, are key regulators of the formation of secondary walls in woody tissues of Arabidopsis. Plant Cell 19, 270-280 (2007).

40. Yang, S. D., Seo, P. J., Yoon, H. K. \& Park, C. M. The Arabidopsis NAC transcription factor VNI2 integrates abscisic acid signals into leaf senescence via the COR/RD genes. Plant Cell 23, 2155-2168 (2011).

41. Uauy, C., Distelfeld, A., Fahima, T., Blechl, A. \& Dubcovsky, J. A NAC gene regulating senescence improves grain protein, zinc, and iron content in wheat. Science 314, 1298-1301 (2006).

42. Nakashima, K. et al. NAC transcription factors in plant abiotic stress responses Biochim. Biophys. Acta. 1819, 97-103 (2012).

43. Iuchi, S. et al. Regulation of drought tolerance by gene manipulation of 9-cis-epoxycarotenoid dioxygenase, a key enzyme in abscisic acid biosynthesis in Arabidopsis. Plant J. 27, 325-333 (2001)

44. Garcia, M. E., Lynch, T., Peeters, J., Snowden, C. \& Finkelstein, R. A small plant-specific protein family of ABI five binding proteins (AFPs) regulates stress response in germinating Arabidopsis seeds and seedlings. Plant Mol. Biol. 67, 643-658 (2008)

45. Nylander, M., Svensson, J., Palva, E. T. \& Welin, B. V. Stress-induced accumulation and tissue-specific localization of dehydrins in Arabidopsis thaliana. Plant Mol. Biol. 45, 263-279 (2001).

46. Nakashima, K. et al. Transcriptional regulation of ABI3- and ABA-responsive genes including $R D 29 B$ and $R D 29 A$ in seeds, germinating embryos, and seedlings of Arabidopsis. Plant Mol. Biol. 60, 51-68 (2006).

47. Nishimura, N. et al. ABA-hypersensitive germination1 encodes a protein phosphatase $2 \mathrm{C}$, an essential component of abscisic acid signaling in Arabidopsis seed. Plant J. 50, 935-949 (2007).

48. Haake, V. et al. Transcription factor CBF4 is a regulator of drought adaptation in Arabidopsis. Plant Physiol. 130, 639-648 (2002).

49. Tran, L. S. et al. Isolation and functional analysis of Arabidopsis stress-inducible NAC transcription factors that bind to a drought-responsive cis-element in the early responsive to dehydration stress 1 promoter. Plant Cell 16, 2481-2498 (2004).

50. $\mathrm{Hu}, \mathrm{H}$. et al. Overexpressing a NAM, ATAF, and CUC (NAC) transcription factor enhances drought resistance and salt tolerance in rice. Proc. Natl Acad. Sci. USA 103, 12987-12992 (2006)

51. Yang, X. et al. Genetic analysis and characterization of a new maize association mapping panel for quantitative trait loci dissection. Theor. Appl. Genet. 121, 417-431 (2010).

52. Gent, J. I. et al. CHH islands: de novo DNA methylation in near-gene chromatin regulation in maize. Genome Res. 23, 628-637 (2013).

53. Gent, J. I. et al. Accessible DNA and relative depletion of $\mathrm{H} 3 \mathrm{~K} 9 \mathrm{me} 2$ at maize loci undergoing RNA-directed DNA methylation. Plant Cell 26, 4903-4917 (2014).

54. Qin, F., Shinozaki, K. \& Yamaguchi-Shinozaki, K. Achievements and challenges in understanding plant abiotic stress responses and tolerance. Plant Cell Physiol. 52, 1569-1582 (2011).

55. Tran, L. S., Nishiyama, R., Yamaguchi-Shinozaki, K. \& Shinozaki, K. Potentia utilization of NAC transcription factors to enhance abiotic stress tolerance in plants by biotechnological approach. GM Crops 1, 32-39 (2010).

56. Blum, A. Effective use of water (EUW) and not water-use efficiency (WUE) is the target of crop yield improvement under drought stress. Field Crops Res. 112, 119-123 (2009)

57. Lisch, D. How important are transposons for plant evolution? Nat. Rev. Genet 14, 49-61 (2013)

58. Yang, N. et al. Genome wide association studies using a new nonparametric model reveal the genetic architecture of 17 agronomic traits in an enlarged maize association panel. PLoS Genet. 10, e1004573 (2014). 
59. Bradbury, P. J. et al. TASSEL: software for association mapping of complex traits in diverse samples. Bioinformatics 23, 2633-2635 (2007).

60. Price, A. L. et al. Principal components analysis corrects for stratification in genome-wide association studies. Nat. Genet. 38, 904-909 (2006).

61. Fu, J. et al. RNA sequencing reveals the complex regulatory network in the maize kernel. Nat. Commun. 4, 2832 (2013).

62. Purcell, S. et al. PLINK: a tool set for whole-genome association and population-based linkage analyses. Am. J. Hum. Genet. 81, 559-575 (2007).

63. Livak, K. J. \& Schmittgen, T. D. Analysis of relative gene expression data using real-time quantitative PCR and the $2^{-\Delta \Delta C T}$ method. Methods 25, 402-408 (2001).

64. He, Y., Michaels, S. D. \& Amasino, R. M. Regulation of flowering time by histone acetylation in Arabidopsis. Science 302, 1751-1754 (2003).

65. Bowler, C. et al. Chromatin techniques for plant cells. Plant J. 39, 776-789 (2004).

66. Qin, F. et al. Arabidopsis DREB2A-interacting proteins function as RING E3 ligases and negatively regulate plant drought stress-responsive gene expression. Plant Cell 20, 1693-1707 (2008).

67. Ryu, M. Y., Cho, S. K. \& Kim, W. T. The Arabidopsis C3H2C3-type RING E3 ubiquitin ligase AtAIRP1 is a positive regulator of an abscisic acid-dependent response to drought stress. Plant Physiol. 154, 1983-1997 (2010).

68. Ishida, Y., Hiei, Y. \& Komari, T. Agrobacterium-mediated transformation of maize. Nat. Protoc. 2, 1614-1621 (2007)

69. Huang, D. W., Sherman, B. T. \& Lempicki, R. A. Systematic and integrative analysis of large gene lists using DAVID bioinformatics resources. Nat. Protoc. 4, 44-57 (2009)

70. Librado, P. \& Rozas, J. DnaSP v5: a software for comprehensive analysis of DNA polymorphism data. Bioinformatics 25, 1451-1452 (2009).

\section{Acknowledgements}

We thank Dr Toshiyuki Komori (Japan Tobacco Inc.) for kindly providing us with the maize transformation plasmid pSBI and pSBII. We thank Professor Yongxiu Liu

(Institute of Botany, Chinese Academy of Sciences) for providing us the Arabidopsis

suvh4-3 mutant seeds. We also thank Ms Suhua Li for her excellent technical support in maize transformation. This research was supported by grants from the National Hi-Tech

Research and Development Program of China (2012AA10A306-4), the National Basic Research Program of China (2012CB114302-4), the Chinese Academy of Sciences (XDA08010206) and the National Natural Science Foundation of China (31471505) to F.Q.

\section{Author contributions}

H.M. performed most of the experiments with the exception of GWAS. H.W. and S.L. carried out the GWAS of maize drought tolerance and identified the ZmNAC111 gene. Z.L. helped with propagation of the materials and genotyping of MITE insertion. X.Y., J.Y. and J.L. provided the maize and teosinte materials, and the SNP information and helpful comments on the work. L.-S.P.T. provided advice on the experiments. F.Q. designed the research and wrote the manuscript.

\section{Additional information}

Accession codes: The RNA sequencing data for this research have been deposited in the NCBI Sequence Read Archive under accession code SRP053172.

Supplementary Information accompanies this paper at http://www.nature.com/ naturecommunications

Competing financial interests: The authors declare no competing financial interests.

Reprints and permission information is available online at http://npg.nature.com/ reprintsandpermissions/

How to cite this article: Mao, H. et al. A transposable element in a NAC gene is associated with drought tolerance in maize seedlings. Nat. Commun. 6:8326 doi: $10.1038 /$ ncomms 9326 (2015)

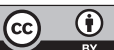
article are included in the article's Creative Commons license, unless indicated otherwise in the credit line; if the material is not included under the Creative Commons license, users will need to obtain permission from the license holder to reproduce the material. To view a copy of this license, visit http://creativecommons.org/licenses/by/4.0/ 